\title{
COUPLED CELL NETWORKS: SEMIGROUPS, LIE ALGEBRAS AND NORMAL FORMS
}

\author{
BOB RINK AND JAN SANDERS
}

\begin{abstract}
We introduce the concept of a semigroup coupled cell network and show that the collection of semigroup network vector fields forms a Lie algebra. This implies that near a dynamical equilibrium the local normal form of a semigroup network is a semigroup network itself. Networks without the semigroup property will support normal forms with a more general network architecture, but these normal forms nevertheless possess the same symmetries and synchronous solutions as the original network. We explain how to compute Lie brackets and normal forms of coupled cell networks and we characterize the SN-decomposition that determines the normal form symmetry. This paper concludes with a generalization to nonhomogeneous networks with the structure of a semigroupoid.
\end{abstract}

\section{INTRODUCTION}

Coupled cell networks appear in many of the sciences and range from crystal models and electrical circuits to numerical discretization schemes, Josephson junction arrays, power grids, the world wide web, ecology, neural networks and systems biology. Not surprisingly, there exists an overwhelming amount of literature on coupled cell networks.

The last decade has seen the development of an extensive mathematical theory of dynamical systems with a network structure; cf. [16], 24, [35], 48, [51. In these network dynamical systems, the evolution of the state of a constituent or "cell" is determined by the states of certain particular other cells. It is generally believed that a network structure has an impact on the behavior of a dynamical system, but it is not always clear how and why.

As an example, let us mention a system of differential equations with a homogeneous coupled cell network structure of the form

$$
\dot{x}_{i}=f\left(x_{\sigma_{1}(i)}, \ldots, x_{\sigma_{n}(i)}\right) \text { for } 1 \leq i \leq N .
$$

These differential equations generate a dynamical system in which the evolution of the variable $x_{i}$ is only determined by the values of $x_{\sigma_{1}(i)}, \ldots, x_{\sigma_{n}(i)}$. The functions

$$
\sigma_{1}, \ldots, \sigma_{n}:\{1, \ldots, N\} \rightarrow\{1, \ldots, N\}
$$

can thus be thought of as a network that prescribes how which cells influence which cells.

The literature on network dynamical systems focuses on the analysis of equilibria, periodic solutions, symmetry, synchrony, structural stability and bifurcations. As in the classical theory of dynamical systems, one often faces the task here of

Received by the editors September 13, 2012 and, in revised form, June 3, 2013.

2010 Mathematics Subject Classification. Primary 37G05.

(C) 2014 American Mathematical Society Reverts to public domain 28 years from publication 
computing a local normal form near a dynamical equilibrium. These normal forms are obtained from coordinate transformations, and in their computation one calculates Lie brackets of vector fields, either implicitly or explicitly. It is here that one encounters an important technical problem:

Differential equations of the form (1.1) in general do not form a Lie algebra.

As a consequence one can not expect that the normal form of a coupled cell network is a coupled cell network as well. This complicates the local analysis and classification of network dynamical systems, because it means that one always has to compute the normal form of a network explicitly to understand its generic behavior - unless one is willing to assume that the network is given in normal form from the beginning; cf. [24, 29. Normal form computations in 13], 21, 26] have revealed that a network structure can have a nontrivial impact on this generic behavior. One wants to understand and predict this.

In this paper, we will formulate an easily verifiable condition on a network structure under which the coupled cell network vector fields do form a Lie subalgebra of the Lie algebra of vector fields. Our main result is the following:

$$
\begin{gathered}
\text { If }\left\{\sigma_{1}, \ldots, \sigma_{n}\right\} \text { is a semigroup, then the differential equations (1.1) form a Lie } \\
\text { algebra. }
\end{gathered}
$$

In this case, the local normal form of (1.1) is also of the form (1.1).

In addition, we show that the Lie bracket of semigroup coupled cell network vector fields can be lifted to a symbolic bracket that only involves the function $f$. Normal form calculations can be performed at this symbolic level, and one only returns to the reality of the differential equation when one is done computing. We also show that the symbolic space carries a dynamics of its own, determined by a certain fundamental network.

This situation is analogous to that of Hamiltonian vector fields, of which the Lie bracket is determined by the Poisson bracket of Hamiltonian functions. As a consequence, Hamiltonian normal forms are usually computed at the level of functions. Moreover, the symbolic dynamics of Hamiltonian functions is determined by a Poisson structure; cf. 41].

When $\sigma_{1}, \ldots, \sigma_{n}$ do not form a semigroup, then we suggest that one simply completes them to the smallest collection

$$
\sigma_{1}, \ldots, \sigma_{n}, \sigma_{n+1}, \ldots, \sigma_{n^{\prime}}:\{1, \ldots, N\} \rightarrow\{1, \ldots, N\}
$$

that does form a semigroup under composition. Then (1.1) can be written as

$$
\dot{x}_{i}=f^{\prime}\left(x_{\sigma_{1}(i)}, \ldots, x_{\sigma_{n^{\prime}}(i)}\right) \text { with } f^{\prime}\left(X_{1}, \ldots, X_{n}, X_{n+1}, \ldots, X_{n^{\prime}}\right):=f\left(X_{1}, \ldots, X_{n}\right) \text {. }
$$

The normal form of (1.1) will now lie within the extended class of semigroup coupled cell networks, there being no guarantee that it is again of the original form (1.1).

Thus one can choose: either to respect any given network structure as if it were a law of nature, so that no normal form can be computed, or to extend every network to a semigroup network and live with the consequences. One can object that even simple networks may need a lot of extension before they form a semigroup. But as an argument in favor of the semigroup approach, let us mention that the symmetries and synchrony spaces of a network are not at all affected by our semigroup extension. This implies in particular that these symmetries and synchrony spaces will also be present in the local normal form of the network. This latter property is both pleasant and important, if only in view of the large 
amount of research that has been devoted to symmetry [5], 6], 9], [18, [25], 31] and synchrony [2, 3], 44, 8], [10, [11, 27], 32], 34], 38, [49], [51], [53] in coupled cell networks. Semigroups may well be the natural invariants of coupled cell networks, even more than groups and symmetries.

Normal forms are computed by applying coordinate transformations [42, 45], [46], 47. These transformations can be in the phase space of a differential equation, but in our case they take place in the space of functions $f$ and have the form of a series expansion

$$
f \mapsto e^{\operatorname{ad}_{g}^{\Sigma}} f=f+\operatorname{ad}_{g}^{\Sigma}(f)+\frac{1}{2}\left(\operatorname{ad}_{g}^{\Sigma}\right)^{2}(f)+\ldots .
$$

Here $f$ is the function to be transformed and normalized, $g$ generates the coordinate transformation and $\mathrm{ad}^{\Sigma}$ denotes a representation, in this case the adjoint representation of the Lie algebra of $f$ 's. Although at first sight this may seem a needlessly complicated way to describe coordinate transformations, this "Lie formalism" allows for a very flexible theory which streamlines both the theory and the computations.

The actual computation of the normal form of the function $f$, and in particular the matter of solving homological equations, will not be entirely standard in the context of networks. Some things remain as in the theory of generic vector fields. For example, we show that the adjoint action of a linear element admits an SNdecomposition that determines a normal form symmetry. Other aspects may not carry through, such as the applicability of the Jacobson-Morozov lemma to characterize the complement of the image of the adjoint action of a nilpotent element [7. This is because the Lie algebra of the linear coupled cell network vector fields need not be reductive.

This paper is organized as follows. After giving a formal definition of a homogeneous coupled cell network in Section 2, we show in Section 3 that semigroups arise naturally in the context of coupled cell networks. In Sections 4 and 5 we prove that semigroup network dynamical systems are closed under taking compositions and Lie brackets. Section 6 explains how to compute the normal form of a network dynamical system, while in Sections 7 and 8 we prove that this normal form inherits both the symmetries and the synchrony spaces of the original network. In Section 9 we investigate the SN-decomposition of a linear coupled cell network vector field. This decomposition determines the normal form symmetry. Section 10 describes the aforementioned fundamental network. In Section 11 we actually compute the normal forms of some simple but interesting coupled cell networks, thus demonstrating that a coupled cell network structure can force anomalous steady state bifurcations. Finally, we show in Section 12 that our theory is also applicable to nonhomogeneous or "colored" networks that display the structure of a semigroupoid.

Issues that we do not touch in this paper but aim to treat in subsequent work include:

1. The development of a linear algebra of semigroup coupled cell systems in order to define for example a "semigroup network Jordan normal form".

2. Application of the results in this paper to semigroup networks that arise in applications, such as feed-forward motifs.

3. Better understanding the relation between semigroups and the well-known "groupoid formalism". The results of this paper mainly apply to networks for which the groupoid is trivial, and it would be interesting to study the impact of nontrivial "input symmetries" on bifurcations and normal forms. 


\section{Homogeneous COUPled CELl NeTwORKS}

We shall be interested in dynamical systems with a coupled cell network structure. Such a structure can be determined in various ways [16, [35, 38, [51, but we choose to describe it here by means of a collection of distinct maps

$$
\Sigma=\left\{\sigma_{1}, \ldots, \sigma_{n}\right\} \text { with } \sigma_{1}, \ldots, \sigma_{n}:\{1, \ldots, N\} \rightarrow\{1, \ldots, N\} .
$$

The collection $\Sigma$ has the interpretation of a network with $1 \leq N<\infty$ cells. Indeed, it defines a directed multigraph with $N$ vertices and precisely $n$ arrows pointing into each vertex, where the arrows pointing towards vertex $1 \leq i \leq N$ emanate from the vertices $\sigma_{1}(i), \ldots, \sigma_{n}(i)$. The number $n$ of incoming arrows per vertex is sometimes called the valence of the network.

In a network dynamical system we think of every vertex $1 \leq i \leq N$ in the network as a cell, of which the state is determined by a variable $x_{i}$ that takes values in a vector space $V$.

Definition 2.1. Let $\Sigma=\left\{\sigma_{1}, \ldots, \sigma_{n}\right\}$ be a collection of $n$ distinct maps on $N$ elements, $V$ a finite-dimensional real vector space and $f: V^{n} \rightarrow V$ a smooth function. Then we define

$$
\gamma_{f}: V^{N} \rightarrow V^{N} \text { by }\left(\gamma_{f}\right)_{i}(x):=f\left(x_{\sigma_{1}(i)}, \ldots, x_{\sigma_{n}(i)}\right) \text { for } 1 \leq i \leq N .
$$

Depending on the context, we will say that $\gamma_{f}$ is a homogeneous coupled cell network map or a homogeneous coupled cell network vector field subject to $\Sigma$.

Remark 2.2. In the literature, $\gamma_{f}$ is also called an admissible map/vector field. These maps and vector fields are commonly defined in terms of the groupoid of input equivalences of the cells in the network; see [35], 38] and [51]. For network maps/vector fields of the form (2.2) the corresponding groupoid is trivial though: every cell $i$ unambiguously has precisely one well-defined $j$-th input. A nontrivial groupoid arises when two or more different inputs of a cell can be interchanged without any effect on the dynamics. This case will be discussed in some detail in Section 8

Dynamical systems with a coupled cell network structure arise when we iterate the map $\gamma_{f}$ or integrate the vector field that it defines. The iterative dynamics on $V^{N}$ has the special property that the state of cell $i$ at time $m+1$ depends only on the states of the cells $\sigma_{1}(i), \ldots, \sigma_{n}(i)$ at time $m$ :

$$
x^{(m+1)}=\gamma_{f}\left(x^{(m)}\right) \text { if and only if } x_{i}^{(m+1)}=f\left(x_{\sigma_{1}(i)}^{(m)}, \ldots, x_{\sigma_{n}(i)}^{(m)}\right) \text { for } 1 \leq i \leq N .
$$

The continuous-time dynamical system on $V^{N}$ displays the same property infinitesimally: it is determined by the ordinary differential equations

$$
\dot{x}=\gamma_{f}(x) \text { if and only if } \dot{x}_{i}=f\left(x_{\sigma_{1}(i)}, \ldots, x_{\sigma_{n}(i)}\right) \text { for } 1 \leq i \leq N .
$$

We aim to understand how the network structure of $\gamma_{f}$ impacts these dynamical systems.

Example 2.3. An example of a directed multigraph is shown in Figure 1, where the number of cells is $N=3$ and the valence is equal to $n=2$. The maps $\sigma_{1}$ and 


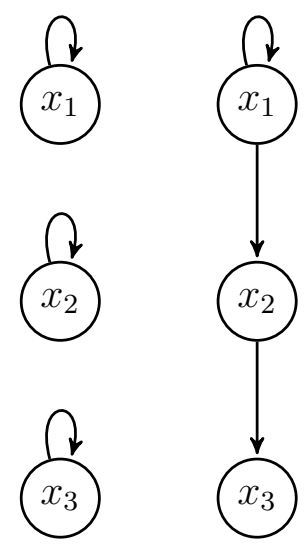

Figure 1. The collection $\left\{\sigma_{1}, \sigma_{2}\right\}$ depicted as a directed multigraph.

$\sigma_{2}$ are given by

$$
\begin{aligned}
& \sigma_{1}(1)=1, \sigma_{1}(2)=2, \sigma_{1}(3)=3, \\
& \sigma_{2}(1)=1, \sigma_{2}(2)=1, \sigma_{2}(3)=2 .
\end{aligned}
$$

A coupled cell network map/vector field subject to $\left\{\sigma_{1}, \sigma_{2}\right\}$ is of the form

$$
\gamma_{f}\left(x_{1}, x_{2}, x_{3}\right)=\left(f\left(x_{1}, x_{1}\right), f\left(x_{2}, x_{1}\right), f\left(x_{3}, x_{2}\right)\right) .
$$

This network has obtained some attention [13, [24, 26, [29, [40] because it supports an anomalous codimension one nilpotent double Hopf bifurcation when $\operatorname{dim} V=2$.

Example 2.4. In this example we let $\sigma_{1}, \sigma_{2}$ be as in Example 2.3 and we also define $\sigma_{3}$ as

$$
\sigma_{3}(1)=1, \sigma_{3}(2)=1, \sigma_{3}(3)=1 .
$$

The network defined by $\left\{\sigma_{1}, \sigma_{2}, \sigma_{3}\right\}$ is depicted in Figure 2 ,

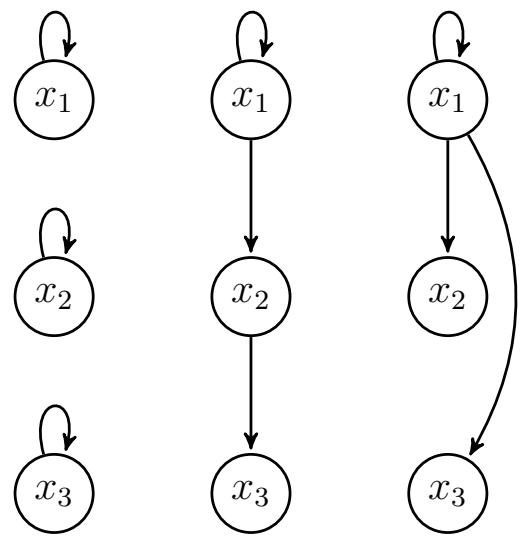

Figure 2. The collection $\left\{\sigma_{1}, \sigma_{2}, \sigma_{3}\right\}$ depicted as a directed multigraph. 
A coupled cell network map/vector field subject to $\left\{\sigma_{1}, \sigma_{2}, \sigma_{3}\right\}$ has the form

$$
\gamma_{g}\left(x_{1}, x_{2}, x_{3}\right)=\left(g\left(x_{1}, x_{1}, x_{1}\right), g\left(x_{2}, x_{1}, x_{1}\right), g\left(x_{3}, x_{2}, x_{1}\right)\right) .
$$

We remark that this example is a generalization of Example 2.3. if $\gamma_{f}$ is as in Example 2.3 and if we define $g\left(X_{1}, X_{2}, X_{3}\right):=f\left(X_{1}, X_{2}\right)$, then $\gamma_{g}=\gamma_{f}$. In other words, (2.5) arises as a special case of (2.6).

\section{Semigroups}

A first and obvious difficulty that arises in the study of coupled cell network dynamical systems is that the composition $\gamma_{f} \circ \gamma_{g}$ of two coupled cell network maps with an identical network structure may not have that same network structure.

Dynamically, this implies for example that the equation $\gamma_{f}(x)=x$ for the steady states of $\gamma_{f}$ and the equation $\left(\gamma_{f}\right)^{m}(x)=x$ for its periodic solutions may have quite a different nature. We illustrate this phenomenon in the following example:

Example 3.1. Again, let $N=3$ and let $\sigma_{1}, \sigma_{2}, \sigma_{3}$ be defined as in Examples 2.3 and 2.4. If

$$
\begin{aligned}
& \gamma_{f}\left(x_{1}, x_{2}, x_{3}\right)=\left(f\left(x_{1}, x_{1}\right), f\left(x_{2}, x_{1}\right), f\left(x_{3}, x_{2}\right)\right), \\
& \gamma_{g}\left(x_{1}, x_{2}, x_{3}\right)=\left(g\left(x_{1}, x_{1}\right), g\left(x_{2}, x_{1}\right), g\left(x_{3}, x_{2}\right)\right)
\end{aligned}
$$

are coupled cell network maps subject to $\left\{\sigma_{1}, \sigma_{2}\right\}$, then the composition

$$
\begin{aligned}
& \left(\gamma_{f} \circ \gamma_{g}\right)\left(x_{1}, x_{2}, x_{3}\right) \\
& \quad=\left(f\left(g\left(x_{1}, x_{1}\right), g\left(x_{1}, x_{1}\right)\right), f\left(g\left(x_{2}, x_{1}\right), g\left(x_{1}, x_{1}\right)\right), f\left(g\left(x_{3}, x_{2}\right), g\left(x_{2}, x_{1}\right)\right)\right)
\end{aligned}
$$

in general is not a coupled cell network map subject to $\left\{\sigma_{1}, \sigma_{2}\right\}$. i.e.

On the other hand, when $\gamma_{f}$ and $\gamma_{g}$ are network maps subject to $\left\{\sigma_{1}, \sigma_{2}, \sigma_{3}\right\}$,

$$
\begin{aligned}
& \gamma_{f}\left(x_{1}, x_{2}, x_{3}\right)=\left(f\left(x_{1}, x_{1}, x_{1}\right), f\left(x_{2}, x_{1}, x_{1}\right), f\left(x_{3}, x_{2}, x_{1}\right)\right), \\
& \gamma_{g}\left(x_{1}, x_{2}, x_{3}\right)=\left(g\left(x_{1}, x_{1}, x_{1}\right), g\left(x_{2}, x_{1}, x_{1}\right), g\left(x_{3}, x_{2}, x_{1}\right)\right),
\end{aligned}
$$

then it holds that

$$
\begin{aligned}
& \left(\gamma_{f} \circ \gamma_{g}\right)_{1}\left(x_{1}, x_{2}, x_{3}\right)=f\left(g\left(x_{1}, x_{1}, x_{1}\right), g\left(x_{1}, x_{1}, x_{1}\right), g\left(x_{1}, x_{1}, x_{1}\right)\right), \\
& \left(\gamma_{f} \circ \gamma_{g}\right)_{2}\left(x_{1}, x_{2}, x_{3}\right)=f\left(g\left(x_{2}, x_{1}, x_{1}\right), g\left(x_{1}, x_{1}, x_{1}\right), g\left(x_{1}, x_{1}, x_{1}\right)\right), \\
& \left(\gamma_{f} \circ \gamma_{g}\right)_{3}\left(x_{1}, x_{2}, x_{3}\right)=f\left(g\left(x_{3}, x_{2}, x_{1}\right), g\left(x_{2}, x_{1}, x_{1}\right), g\left(x_{1}, x_{1}, x_{1}\right)\right) .
\end{aligned}
$$

This demonstrates that $\gamma_{f} \circ \gamma_{g}$ is also a coupled cell network map subject to $\left\{\sigma_{1}, \sigma_{2}, \sigma_{3}\right\}$. Indeed, $\gamma_{f} \circ \gamma_{g}=\gamma_{h}$, where

$$
h\left(X_{1}, X_{2}, X_{3}\right)=f\left(g\left(X_{1}, X_{2}, X_{3}\right), g\left(X_{2}, X_{3}, X_{3}\right), g\left(X_{3}, X_{3}, X_{3}\right)\right) .
$$

To understand when, in general, the composition of two coupled cell network maps is again a coupled cell network map, we compute that

$$
\left(\gamma_{f} \circ \gamma_{g}\right)_{i}(x)=f\left(\ldots,\left(\gamma_{g}\right)_{\sigma_{j}(i)}(x), \ldots\right)=f\left(\ldots, g\left(x_{\sigma_{1}\left(\sigma_{j}(i)\right)}, \ldots, x_{\sigma_{n}\left(\sigma_{j}(i)\right)}\right), \ldots\right) .
$$


The right hand side of (3.7) is an $i$-independent function of $\left(x_{\sigma_{1}(i)}, \ldots, x_{\sigma_{n}(i)}\right)$ precisely when for all $1 \leq j_{1}, j_{2} \leq n$ and all $1 \leq i \leq N$ it holds that $\sigma_{j_{1}}\left(\sigma_{j_{2}}(i)\right)=$ $\sigma_{j_{3}}(i)$ for some $1 \leq j_{3} \leq n$. In other words, $\gamma_{f} \circ \gamma_{g}$ is a coupled cell network map when $\Sigma$ is a semigroup:

Definition 3.2. We say that $\Sigma=\left\{\sigma_{1}, \ldots, \sigma_{n}\right\}$ is a semigroup if for all $1 \leq j_{1}, j_{2} \leq$ $n$ there is a unique $1 \leq j_{3} \leq n$ such that $\sigma_{j_{1}} \circ \sigma_{j_{2}}=\sigma_{j_{3}}$.

Viewing $\Sigma$ as a directed multigraph, the condition that it is a semigroup just means that this directed multigraph is closed under the backward concatenation of arrows.

Of course, an arbitrary collection $\Sigma=\left\{\sigma_{1}, \ldots, \sigma_{n}\right\}$ need not be a semigroup. Even so, $\Sigma$ generates a unique smallest semigroup

$$
\Sigma^{\prime}=\left\{\sigma_{1}, \ldots, \sigma_{n}, \sigma_{n+1}, \ldots, \sigma_{n^{\prime}}\right\} \text { that contains } \Sigma .
$$

It is clear that every coupled cell network map $\gamma_{f}$ subject to $\Sigma$ is also a coupled cell network map subject to the semigroup $\Sigma^{\prime}$. Indeed, if we define

$$
f^{\prime}\left(X_{1}, \ldots, X_{n}, X_{n+1}, \ldots, X_{n^{\prime}}\right):=f\left(X_{1}, \ldots, X_{n}\right),
$$

then it obviously holds that

$$
\begin{aligned}
\left(\gamma_{f^{\prime}}\right)_{i}(x) & =f^{\prime}\left(x_{\sigma_{1}(i)}, \ldots, x_{\sigma_{n}(i)}, x_{\sigma_{n+1}(i)}, \ldots, x_{\sigma_{n^{\prime}}(i)}\right) \\
& =f\left(x_{\sigma_{1}(i)}, \ldots, x_{\sigma_{n}(i)}\right)=\left(\gamma_{f}\right)_{i}(x) .
\end{aligned}
$$

We thus propose to augment $\Sigma$ to the semigroup $\Sigma^{\prime}$ and to think of every coupled cell network map subject to $\Sigma$ as a (special case of a) coupled cell network map subject to $\Sigma^{\prime}$.

Example 3.3. Again, let $N=3$ and let $\sigma_{1}, \sigma_{2}, \sigma_{3}$ be defined as in Examples 2.3 and 2.4. It holds that $\sigma_{2}^{2}=\sigma_{3}$, so the collection $\left\{\sigma_{1}, \sigma_{2}\right\}$ is not a semigroup. On the other hand, one quickly computes that the composition table of $\left\{\sigma_{1}, \sigma_{2}, \sigma_{3}\right\}$ is given by

\begin{tabular}{c|ccc}
$\circ$ & $\sigma_{1}$ & $\sigma_{2}$ & $\sigma_{3}$ \\
\hline$\sigma_{1}$ & $\sigma_{1}$ & $\sigma_{2}$ & $\sigma_{3}$ \\
$\sigma_{2}$ & $\sigma_{2}$ & $\sigma_{3}$ & $\sigma_{3}$ \\
$\sigma_{3}$ & $\sigma_{3}$ & $\sigma_{3}$ & $\sigma_{3}$
\end{tabular}.

This shows that $\left\{\sigma_{1}, \sigma_{2}, \sigma_{3}\right\}$ is closed under composition and hence is the smallest semigroup containing $\left\{\sigma_{1}, \sigma_{2}\right\}$.

\section{Composition OF NeTWORK MAPS}

To better understand how network maps behave under composition and in order to simplify our notation, let us define the maps

$$
\pi_{i}: V^{N} \rightarrow V^{n} \text { by } \pi_{i}\left(x_{1}, \ldots, x_{N}\right):=\left(x_{\sigma_{1}(i)}, \ldots, x_{\sigma_{n}(i)}\right) \text { for } 1 \leq i \leq N .
$$

This definition allows us to write (2.2) simply as

$$
\left(\gamma_{f}\right)_{i}:=f \circ \pi_{i} \text {. }
$$

Expression (3.7) moreover turns into the formula

$$
\left(\gamma_{f} \circ \gamma_{g}\right)_{i}=f \circ\left(g \circ \pi_{\sigma_{1}(i)} \times \ldots \times g \circ \pi_{\sigma_{n}(i)}\right) .
$$

The following technical result helps us write the right hand side of (4.9) in the form $h \circ \pi_{i}$ for some function $h: V^{n} \rightarrow V$, whenever $\Sigma$ is a semigroup. 
Theorem 4.1. Let $\Sigma=\left\{\sigma_{1}, \ldots, \sigma_{n}\right\}$ be a semigroup. Then for all $1 \leq j \leq n$ there exists a linear map $A_{\sigma_{j}}: V^{n} \rightarrow V^{n}$ with the property that

$$
A_{\sigma_{j}} \circ \pi_{i}=\pi_{\sigma_{j}(i)} \text { for all } 1 \leq i \leq N \text { and all } 1 \leq j \leq n .
$$

Moreover, it holds that $A_{\sigma_{j_{1}}} \circ A_{\sigma_{j_{2}}}=A_{\sigma_{j_{1}} \circ \sigma_{j_{2}}}$ for all $1 \leq j_{1}, j_{2} \leq n$.

Proof. Because $\Sigma$ is a semigroup, we can associate to each map $\sigma_{j} \in \Sigma$ a unique map

$$
\widetilde{\sigma}_{j}:\{1, \ldots, n\} \rightarrow\{1, \ldots, n\} \text { defined via the formula } \sigma_{\widetilde{\sigma}_{j}(k)}=\sigma_{j} \circ \sigma_{k} .
$$

We now define the map $A_{\sigma_{j}}: V^{n} \rightarrow V^{n}$ as

$$
A_{\sigma_{j}}\left(X_{1}, \ldots, X_{n}\right):=\left(X_{\widetilde{\sigma}_{1}(j)}, \ldots, X_{\widetilde{\sigma}_{n}(j)}\right) .
$$

With this definition it holds that

$$
\begin{aligned}
\left(A_{\sigma_{j}} \circ \pi_{i}\right)(x) & =A_{\sigma_{j}}\left(x_{\sigma_{1}(i)}, \ldots, x_{\sigma_{n}(i)}\right)=\left(x_{\sigma_{\widetilde{\sigma}_{1}(j)}(i)}, \ldots, x_{\sigma_{\widetilde{\sigma}_{n}(j)}(i)}\right) \\
& =\left(x_{\sigma_{1}\left(\sigma_{j}(i)\right)}, \ldots, x_{\sigma_{n}\left(\sigma_{j}(i)\right)}\right)=\pi_{\sigma_{j}(i)}(x) .
\end{aligned}
$$

Remarking moreover that

$$
\sigma_{\widetilde{\sigma}_{\widetilde{\sigma}_{k}\left(j_{1}\right)}\left(j_{2}\right)}=\sigma_{\widetilde{\sigma}_{k}\left(j_{1}\right)} \circ \sigma_{j_{2}}=\sigma_{k} \circ \sigma_{j_{1}} \circ \sigma_{j_{2}}=\sigma_{k} \circ \sigma_{\widetilde{\sigma}_{j_{1}}\left(j_{2}\right)}=\sigma_{\widetilde{\sigma}_{k}\left(\widetilde{\sigma}_{j_{1}}\left(j_{2}\right)\right)},
$$

and hence that $\widetilde{\sigma}_{\widetilde{\sigma}_{k}\left(j_{1}\right)}\left(j_{2}\right)=\widetilde{\sigma}_{k}\left(\widetilde{\sigma}_{j_{1}}\left(j_{2}\right)\right)$ for all $1 \leq k \leq n$, we also find that

$$
\begin{aligned}
& \left(A_{\sigma_{j_{1}}} \circ A_{\sigma_{j_{2}}}\right)\left(X_{1}, \ldots, X_{n}\right)=A_{\sigma_{j_{1}}}\left(X_{\widetilde{\sigma}_{1}\left(j_{2}\right)}, \ldots, X_{\widetilde{\sigma}_{n}\left(j_{2}\right)}\right) \\
& =\left(X_{\widetilde{\sigma}_{\widetilde{\sigma}_{1}\left(j_{1}\right)}\left(j_{2}\right)}, \ldots, X_{\widetilde{\sigma}_{\widetilde{\sigma}_{n}\left(j_{1}\right)}\left(j_{2}\right)}\right)=\left(X_{\widetilde{\sigma}_{1}\left(\widetilde{\sigma}_{j_{1}}\left(j_{2}\right)\right)}, \ldots, X_{\widetilde{\sigma}_{n}\left(\widetilde{\sigma}_{j_{1}}\left(j_{2}\right)\right)}\right) \\
& =A_{\sigma_{\widetilde{\sigma}_{j_{1}}\left(j_{2}\right)}}\left(X_{1}, \ldots, X_{n}\right)=A_{\sigma_{j_{1}} \circ \sigma_{j_{2}}}\left(X_{1}, \ldots, X_{n}\right) .
\end{aligned}
$$

This proves the theorem.

The identity $A_{\sigma_{j_{1}}} \circ A_{\sigma_{j_{2}}}=A_{\sigma_{j_{1}} \circ \sigma_{j_{2}}}$ expresses that the $A_{\sigma_{j}}$ form a representation of the semigroup $\Sigma$. Using this representation we obtain:

Theorem 4.2. Let $\Sigma=\left\{\sigma_{1}, \ldots, \sigma_{n}\right\}$ be a semigroup. Define for $f, g: V^{n} \rightarrow V$ the function

$$
f \circ_{\Sigma} g: V^{n} \rightarrow V \text { by } f \circ_{\Sigma} g:=f \circ\left(\left(g \circ A_{\sigma_{1}}\right) \times \ldots \times\left(g \circ A_{\sigma_{n}}\right)\right) .
$$

Then

$$
\gamma_{f} \circ \gamma_{g}=\gamma_{f \circ \Sigma} \cdot
$$

Proof. From formula (4.9) and Theorem 4.1 .

Theorem 4.2 reveals once more that if $\Sigma$ is a semigroup, then the composition of two coupled cell network maps $\gamma_{f}$ and $\gamma_{g}$ is again a coupled cell network map, namely $\gamma_{f \circ \Sigma} g$. More importantly, it shows how to compute $f \circ_{\Sigma} g$ "symbolically", i.e. using only the functions $f$ and $g$ and a representation of the network semigroup.

The final result of this section ensures that the "symbolic composition" $o_{\Sigma}$ makes the space $C^{\infty}\left(V^{n}, V\right)$ into an associative algebra.

\section{Lemma 4.3.}

$$
\left(f \circ_{\Sigma} g\right) \circ_{\Sigma} h=f \circ_{\Sigma}\left(g \circ_{\Sigma} h\right)
$$


Proof.

$$
\begin{aligned}
& \left(f \circ_{\Sigma} g\right) \circ_{\Sigma} h(X)=\left(f \circ_{\Sigma} g\right)\left(\ldots, h\left(A_{\sigma_{k}} X\right), \ldots\right)=f\left(\ldots, g\left(A_{\sigma_{j}}\left(\ldots, h\left(A_{\sigma_{k}} X\right), \ldots\right)\right), \ldots\right) \\
& \quad=f\left(\ldots, g\left(\ldots, h\left(A_{\sigma_{\widetilde{\sigma}_{k}(j)}} X\right), \ldots\right)\right)=f\left(\ldots, g\left(\ldots, h\left(A_{\sigma_{k} \circ \sigma_{j}} X\right), \ldots\right)\right) \\
& \quad=f\left(\ldots, g\left(\ldots, h\left(A_{\sigma_{k}} A_{\sigma_{j}} X\right), \ldots\right), \ldots\right)=f\left(\ldots,\left(g \circ_{\Sigma} h\right)\left(A_{\sigma_{j}} X\right), \ldots\right) \\
& \quad=f \circ_{\Sigma}\left(g \circ_{\Sigma} h\right)(X) .
\end{aligned}
$$

With Lemma 4.3 at hand, Theorem 4.2 just means that the linear map

$$
\gamma: C^{\infty}\left(V^{n}, V\right) \rightarrow C^{\infty}\left(V^{N}, V^{N}\right) \text { that sends } f \text { to } \gamma_{f}
$$

is a homomorphism of associative algebras.

Example 4.4. Again, let $N=3$ and let $\sigma_{1}, \sigma_{2}, \sigma_{3}$ be defined as in Examples 2.3 and 2.4. We recall that the composition table of $\left\{\sigma_{1}, \sigma_{2}, \sigma_{3}\right\}$ was given in Example 3.3. The rows of this table express that

$$
\begin{aligned}
& \tilde{\sigma}_{1}(1)=1, \widetilde{\sigma}_{1}(2)=2, \widetilde{\sigma}_{1}(3)=3, \\
& \tilde{\sigma}_{2}(1)=2, \widetilde{\sigma}_{2}(2)=3, \widetilde{\sigma}_{2}(3)=3, \\
& \tilde{\sigma}_{3}(1)=3, \widetilde{\sigma}_{3}(2)=3, \widetilde{\sigma}_{3}(3)=3 .
\end{aligned}
$$

This implies in particular that

$$
\begin{aligned}
& A_{\sigma_{1}}\left(X_{1}, X_{2}, X_{3}\right)=\left(X_{1}, X_{2}, X_{3}\right), \\
& A_{\sigma_{2}}\left(X_{1}, X_{2}, X_{3}\right)=\left(X_{2}, X_{3}, X_{3}\right), \\
& A_{\sigma_{3}}\left(X_{1}, X_{2}, X_{3}\right)=\left(X_{3}, X_{3}, X_{3}\right) .
\end{aligned}
$$

Substitution in (4.13) therefore yields that

$$
f \circ_{\Sigma} g\left(X_{1}, X_{2}, X_{3}\right)=f\left(g\left(X_{1}, X_{2}, X_{3}\right), g\left(X_{2}, X_{3}, X_{3}\right), g\left(X_{3}, X_{3}, X_{3}\right)\right) .
$$

We conclude that $f \circ_{\Sigma} g$ equals the function $h$ found in Example 3.1 .

Remark 4.5. The defining relation

$$
\sigma_{\widetilde{\sigma}_{j}(k)}=\sigma_{j} \circ \sigma_{k} \text { for } \widetilde{\sigma}_{j}:\{1, \ldots, n\} \rightarrow\{1, \ldots, n\}
$$

expresses that the map $\widetilde{\sigma}_{j}$ describes the left-multiplicative behavior of $\sigma_{j}$. The computation

$$
\sigma_{\sigma_{j_{1} \circ \sigma_{j_{2}}}(k)}=\sigma_{j_{1}} \circ \sigma_{j_{2}} \circ \sigma_{k}=\sigma_{j_{1}} \circ \sigma_{\widetilde{\sigma}_{j_{2}}(k)}=\sigma_{\widetilde{\sigma}_{j_{1}}\left(\widetilde{\sigma}_{j_{2}}(k)\right)}
$$

moreover reveals that

$$
\widetilde{\sigma_{j_{1}} \circ \sigma_{j_{2}}}=\widetilde{\sigma}_{j_{1}} \circ \widetilde{\sigma}_{j_{2}} \text { for all } 1 \leq j_{1}, j_{2} \leq n .
$$

This means in particular that the collection $\left\{\widetilde{\sigma}_{1}, \ldots, \widetilde{\sigma}_{n}\right\}$ is closed under composition.

The maps $\widetilde{\sigma}_{1}, \ldots, \widetilde{\sigma}_{n}$ will play an interesting role in this paper. In fact, we will show in Section 10 that they are themselves the network maps of a certain "fundamental network" that fully determines the fate of all network dynamical systems subject to $\Sigma$. 
Remark 4.6. For a map $\sigma:\{1, \ldots, n\} \rightarrow\{1, \ldots, m\}$ let us denote by $\lambda_{\sigma}: V^{m} \rightarrow V^{n}$ the linear map

$$
\lambda_{\sigma}\left(X_{1}, \ldots, X_{m}\right):=\left(X_{\sigma(1)}, \ldots, X_{\sigma(n)}\right) .
$$

This means that the matrix of the map $\lambda_{\sigma}$ has precisely one $\mathrm{id}_{V}$ on each row and zeroes elsewhere. We will denote the space of such maps by

$$
\Lambda(m, n):=\left\{\lambda_{\sigma}: V^{m} \rightarrow V^{n} \mid \sigma:\{1, \ldots, n\} \rightarrow\{1, \ldots, m\}\right\} .
$$

One quickly checks that the assignment $\lambda: \sigma \mapsto \lambda_{\sigma}$ is contravariant. More precisely, if $\sigma:\{1, \ldots, n\} \rightarrow\{1, \ldots, m\}$ and $\tau:\{1, \ldots, m\} \rightarrow\{1, \ldots, l\}$, then

$$
\lambda_{\sigma} \circ \lambda_{\tau}=\lambda_{\tau \circ \sigma} \text {. }
$$

In particular, $\Lambda(m, m)$ is a semigroup and the map $\sigma \mapsto \lambda_{\sigma}$ an anti-homomorphism from the semigroup of all maps from $\{1, \ldots, m\}$ to itself to the semigroup $\Lambda(m, m)$.

The maps $\pi_{i}$ and $A_{\sigma_{j}}$ defined above are examples of such maps:

$$
\begin{aligned}
\pi_{i} & =\lambda_{\sigma^{i}} \text { with } \sigma^{i}:\{1, \ldots, n\} \rightarrow\{1, \ldots, N\} \text { defined as } \sigma^{i}(j):=\sigma_{j}(i), \\
A_{\sigma_{j}} & =\lambda_{\widetilde{\sigma}^{j}} \text { with } \tilde{\sigma}^{j}:\{1, \ldots, n\} \rightarrow\{1, \ldots, n\} \text { defined by } \widetilde{\sigma}^{j}(k):=\widetilde{\sigma}_{k}(j) .
\end{aligned}
$$

This observation can be used give a proof of Theorem 4.1 that is free of coordinates:

Proof of Theorem 4.1 without coordinates. We observe that

$$
\sigma^{i}\left(\widetilde{\sigma}^{j}(k)\right)=\sigma^{i}\left(\widetilde{\sigma}_{k}(j)\right)=\sigma_{\widetilde{\sigma}_{k}(j)}(i)=\left(\sigma_{k} \circ \sigma_{j}\right)(i)=\sigma_{k}\left(\sigma_{j}(i)\right)=\sigma^{\sigma_{j}(i)}(k)
$$

and hence that $\sigma^{i} \circ \widetilde{\sigma}^{j}=\sigma^{\sigma_{j}(i)}$. Using this, we find that

$$
A_{\sigma_{j}} \circ \pi_{i}=\lambda_{\widetilde{\sigma}^{j}} \circ \lambda_{\sigma^{i}}=\lambda_{\sigma^{i} \circ \widetilde{\sigma}^{j}}=\lambda_{\sigma^{\sigma_{j}(i)}}=\pi_{\sigma_{j}(i)} .
$$

Similarly, the computation

$$
\begin{gathered}
\sigma_{\left(\widetilde{\sigma}^{j_{2}} \circ \widetilde{\sigma}^{j_{1}}\right)(k)}=\sigma_{\widetilde{\sigma}^{j_{2}}\left(\widetilde{\sigma}^{j_{1}}(k)\right)}=\sigma_{\widetilde{\sigma}^{j_{2}}\left(\widetilde{\sigma}_{k}\left(j_{1}\right)\right)}=\sigma_{\widetilde{\sigma}_{\widetilde{\sigma}_{k}\left(j_{1}\right)}\left(j_{2}\right)} \\
=\sigma_{\widetilde{\sigma}_{k}\left(j_{1}\right)} \circ \sigma_{j_{2}}=\sigma_{k} \circ \sigma_{j_{1}} \circ \sigma_{j_{2}}=\sigma_{k} \circ \sigma_{\widetilde{\sigma}_{j_{1}}\left(j_{2}\right)}=\sigma_{\widetilde{\sigma}_{k}\left(\widetilde{\sigma}_{j_{1}}\left(j_{2}\right)\right)}=\sigma_{\widetilde{\sigma}_{\sigma_{j_{1}}\left(j_{2}\right)}(k)}
\end{gathered}
$$

reveals that

$$
\widetilde{\sigma}^{j_{2}} \circ \widetilde{\sigma}^{j_{1}}=\widetilde{\sigma}^{\widetilde{\sigma}_{j_{1}}\left(j_{2}\right)}
$$

As a consequence,

$$
A_{\sigma_{j_{1}}} \circ A_{\sigma_{j_{2}}}=\lambda_{\widetilde{\sigma}^{j_{1}}} \circ \lambda_{\widetilde{\sigma}^{j_{2}}}=\lambda_{\widetilde{\sigma}^{j_{2} \circ \widetilde{\sigma}^{j_{1}}}}=\lambda_{\widetilde{\sigma}^{\widetilde{\sigma}_{1}\left(j_{2}\right)}}=A_{\sigma_{\widetilde{\sigma}_{j_{1}}\left(j_{2}\right)}}=A_{\sigma_{j_{1}} \circ \sigma_{j_{2}}} .
$$

Unfortunately, this coordinate free proof of Theorem 4.1 is relatively long.

\section{A COUPLED CELL NETWORK BRACKET}

We will now think of $\gamma_{f}: V^{N} \rightarrow V^{N}$ as a vector field that generates the differential equation

$$
\dot{x}=\gamma_{f}(x) .
$$

We suggestively denote by $e^{t \gamma_{f}}$ the time- $t$ flow of the vector field $\gamma_{f}$ on $V^{N}$ and by $\left(e^{t \gamma_{g}}\right)_{*} \gamma_{f}$ the pushforward of the vector field $\gamma_{f}$ under the time- $t$ flow of $\gamma_{g}$. We recall that the Lie bracket of $\gamma_{f}$ and $\gamma_{g}$ is then the vector field $\left[\gamma_{f}, \gamma_{g}\right]: V^{N} \rightarrow V^{N}$ defined as

$$
\left[\gamma_{f}, \gamma_{g}\right](x):=\left.\frac{d}{d t}\right|_{t=0}\left(e^{t \gamma_{f}}\right)_{*} \gamma_{g}=D \gamma_{f}(x) \cdot \gamma_{g}(x)-D \gamma_{g}(x) \cdot \gamma_{f}(x)
$$


The main result of this section is that if $\Sigma$ is a semigroup, then the collection of coupled cell network vector fields is closed under taking Lie brackets.

Theorem 5.1. Let $\Sigma=\left\{\sigma_{1}, \ldots, \sigma_{n}\right\}$ be a semigroup and let the $A_{\sigma_{j}}: V^{n} \rightarrow V^{n}$ be as in Theorem 4.1. Define, for $f, g: V^{n} \rightarrow V$, the function $[f, g]_{\Sigma}: V^{n} \rightarrow V$ by

$$
[f, g]_{\Sigma}:=\sum_{j=1}^{n} D_{j} f \cdot\left(g \circ A_{\sigma_{j}}\right)-D_{j} g \cdot\left(f \circ A_{\sigma_{j}}\right) .
$$

Then

$$
\left[\gamma_{f}, \gamma_{g}\right]=\gamma_{[f, g]_{\Sigma}}
$$

Proof. We start by remarking that

$$
\begin{aligned}
\gamma_{f} & \left(x+t \gamma_{g}(x)\right)_{i}=f\left(\ldots, x_{\sigma_{j}(i)}+t \gamma_{g}(x)_{\sigma_{j}(i)}, \ldots\right) \\
& =f\left(\ldots, x_{\sigma_{j}(i)}+t g\left(x_{\sigma_{1}\left(\sigma_{j}(i)\right)}, \ldots, x_{\sigma_{n}\left(\sigma_{j}(i)\right)}\right), \ldots\right) \\
& =f\left(\ldots, x_{\sigma_{j}(i)}+t g\left(A_{\sigma_{j}}\left(x_{\sigma_{1}(i)}, \ldots, x_{\sigma_{n}(i)}\right)\right), \ldots\right) .
\end{aligned}
$$

Differentiating this identity with respect to $t$ and evaluating the result at $t=0$ gives that

$$
\left(D \gamma_{f}(x) \cdot \gamma_{g}(x)\right)_{i}=\sum_{j=1}^{n} D_{j} f\left(x_{\sigma_{1}(i)}, \ldots, x_{\sigma_{n}(i)}\right) g\left(A_{\sigma_{j}}\left(x_{\sigma_{1}(i)}, \ldots, x_{\sigma_{n}(i)}\right)\right) .
$$

This proves that $\left(D \gamma_{f} \cdot \gamma_{g}\right)_{i}=\left(\sum_{j=1}^{n} D_{j} f \cdot\left(g \circ A_{\sigma_{j}}\right)\right) \circ \pi_{i}$, and hence that $D \gamma_{f} \cdot \gamma_{g}$ is a coupled cell network vector field. With a similar computation for $D \gamma_{g} \cdot \gamma_{f}$, we thus find that the Lie bracket between $\gamma_{f}$ and $\gamma_{g}$ is given by

$$
\begin{aligned}
\left.\gamma_{f}, \gamma_{g}\right]_{i} & =\left(D \gamma_{f} \cdot \gamma_{g}\right)_{i}-\left(D \gamma_{g} \cdot \gamma_{f}\right)_{i}=\sum_{j=1}^{n}\left[D_{j} f \cdot\left(g \circ A_{\sigma_{j}}\right)-D_{j} g \cdot\left(f \circ A_{\sigma_{j}}\right)\right] \circ \pi_{i} \\
& =[f, g]_{\Sigma} \circ \pi_{i} .
\end{aligned}
$$

This proves the theorem.

Lemma 5.2 below states that the "symbolic bracket" $[\cdot, \cdot]_{\Sigma}$ is a Lie bracket.

Lemma 5.2. The bracket $[\cdot, \cdot]_{\Sigma}$ makes $C^{\infty}\left(V^{n}, V\right)$ a Lie algebra. Moreover, the linear map

$\gamma: C^{\infty}\left(V^{n}, V\right) \rightarrow C^{\infty}\left(V^{N}, V^{N}\right)$ that sends $f$ to $\gamma_{f}$ is a Lie algebra homomorphism.

Proof. Anti-symmetry of $[\cdot, \cdot]_{\Sigma}$ is clear from formula (5.15]). The Jacobi identity

$$
\left[f,[g, h]_{\Sigma}\right]_{\Sigma}+\left[g,[h, f]_{\Sigma}\right]_{\Sigma}+\left[h,[f, g]_{\Sigma}\right]_{\Sigma}=0
$$

follows from a somewhat lengthy computation as follows. First of all, because

$$
A_{\sigma_{j}}\left(\ldots, h\left(A_{\sigma_{k}} X\right), \ldots\right)=\left(\ldots, h\left(A_{\sigma_{\widetilde{\sigma}_{k}(j)}} X\right), \ldots\right)=\left(\ldots, h\left(A_{\sigma_{k}} A_{\sigma_{j}} X\right), \ldots\right),
$$

we find that

$$
\begin{gathered}
\left(g \circ A_{\sigma_{j}}\right)\left(X+t\left(\ldots, h\left(A_{\sigma_{k}} X\right), \ldots\right)\right)=g\left(A_{\sigma_{j}} X+t A_{\sigma_{j}}\left(\ldots, h\left(A_{\sigma_{k}} X\right), \ldots\right)\right) \\
=g\left(A_{\sigma_{j}} X+t\left(\ldots, h\left(A_{\sigma_{k}} A_{\sigma_{j}} X\right), \ldots\right)\right) .
\end{gathered}
$$


Differentiating this identity with respect to $t$ and evaluating the result at $t=0$ yields that

$$
\sum_{k=1}^{n} D_{k}\left(g \circ A_{\sigma_{j}}\right) \cdot\left(h \circ A_{\sigma_{k}}\right)=\sum_{k=1}^{n}\left(D_{k} g \circ A_{\sigma_{j}}\right) \cdot\left(h \circ A_{\sigma_{k}} \circ A_{\sigma_{j}}\right) .
$$

With this in mind, we now compute that

$$
\begin{aligned}
{\left[f,[g, h]_{\Sigma}\right]_{\Sigma}=} & \sum_{j=1}^{n} D_{j} f \cdot\left([g, h]_{\Sigma} \circ A_{\sigma_{j}}\right)-D_{j}[g, h]_{\Sigma} \cdot\left(f \circ A_{\sigma_{j}}\right) \\
=\sum_{j, k=1}^{n} \quad D_{j} f \cdot\left(D_{k} g \circ A_{\sigma_{j}}\right) \cdot\left(h \circ A_{\sigma_{k}} \circ A_{\sigma_{j}}\right) & \quad-D_{j} f \cdot\left(D_{k} h \circ A_{\sigma_{j}}\right) \cdot\left(g \circ A_{\sigma_{k}} \circ A_{\sigma_{j}}\right) \\
& -D_{k} g \cdot D_{j}\left(h \circ A_{\sigma_{k}}\right) \cdot\left(f \circ A_{\sigma_{j}}\right)+D_{k} h \cdot D_{j}\left(g \circ A_{\sigma_{k}}\right) \cdot\left(f \circ A_{\sigma_{j}}\right) \\
& -D_{j, k}^{2} g \cdot\left(h \circ A_{\sigma_{k}}, f \circ A_{\sigma_{j}}\right)+D_{j, k}^{2} h \cdot\left(g \circ A_{\sigma_{k}}, f \circ A_{\sigma_{j}}\right)
\end{aligned}
$$

Using the symmetry of the second derivatives, the Jacobi identity follows from cyclically permuting $f, g$ and $h$ in the above expression and summing the results. This proves that $C^{\infty}\left(V^{n}, V\right)$ is a Lie algebra. Theorem 5.1 means that $\gamma$ is a Lie algebra homomorphism.

Example 5.3. Again, let $N=3$ and let $\sigma_{1}, \sigma_{2}, \sigma_{3}$ be defined as in Examples 2.3 and 2.4. We recall that $A_{\sigma_{1}}, A_{\sigma_{2}}$ and $A_{\sigma_{3}}$ were computed in Example 4.4. It follows that

$$
\begin{aligned}
{[f, g]_{\Sigma}(X) } & =D_{1} f\left(X_{1}, X_{2}, X_{3}\right) \cdot g\left(X_{1}, X_{2}, X_{3}\right)+D_{2} f\left(X_{1}, X_{2}, X_{3}\right) \cdot g\left(X_{2}, X_{3}, X_{3}\right) \\
& +D_{3} f\left(X_{1}, X_{2}, X_{3}\right) \cdot g\left(X_{3}, X_{3}, X_{3}\right)-D_{1} g\left(X_{1}, X_{2}, X_{3}\right) \cdot f\left(X_{1}, X_{2}, X_{3}\right) \\
& -D_{2} g\left(X_{1}, X_{2}, X_{3}\right) \cdot f\left(X_{2}, X_{3}, X_{3}\right)-D_{3} g\left(X_{1}, X_{2}, X_{3}\right) \cdot f\left(X_{3}, X_{3}, X_{3}\right) .
\end{aligned}
$$

\section{COUPLED CELl NETWORK NORMAL FORMS}

Normal forms are an essential tool in the study of the dynamics and bifurcations of maps and vector fields near equilibria; cf. [42, 47. In this section we will show that it can be arranged that the normal form of a semigroup coupled cell network is a coupled cell network as well. This normal form can moreover be computed "symbolically", i.e. at the level of the function $f$. With Theorem 5.1 at hand, this result is perhaps to be expected. We nevertheless state two illustrative theorems in this section and sketch their proofs.

We start by making a few standard definitions. First of all, we define for $f \in$ $C^{\infty}\left(V^{n}, V\right)$ the operator $\operatorname{ad}_{f}^{\Sigma}: C^{\infty}\left(V^{n}, V\right) \rightarrow C^{\infty}\left(V^{n}, V\right)$ by

$$
\operatorname{ad}_{f}^{\Sigma}(g):=[f, g]_{\Sigma} .
$$

Next, we define for every $k=0,1,2, \ldots$ the finite-dimensional subspace

$$
P^{k}:=\left\{f: V^{n} \rightarrow V \text { homogeneous polynomial of degree } k+1\right\} \subset C^{\infty}\left(V^{n}, V\right) .
$$


One can observe that $P^{0}=L\left(V^{N}, V\right)$ and that if $f \in P^{k}$ and $g \in P^{l}$, then $[f, g]_{\Sigma} \in P^{k+l}$, as is obvious from formula (5.15). In particular, we have that

$$
\text { if } f_{0} \in L\left(V^{n}, V\right) \text {, then } \operatorname{ad}_{f_{0}}^{\Sigma}: P^{k} \rightarrow P^{k} \text {. }
$$

With this in mind, we formulate the first main result of this section. It essentially states that one may restrict the study of semigroup coupled cell networks near local equilibria to semigroup coupled cell networks of a very specific "normal form".

Theorem 6.1 (Coupled cell network normal form theorem). Let $\Sigma=\left\{\sigma_{1}, \ldots, \sigma_{n}\right\}$ be a semigroup, $f \in C^{\infty}\left(V^{n}, V\right)$ and assume that $f(0)=0$. We Taylor expand $f$ as

$$
f=f_{0}+f_{1}+f_{2}+\ldots \text { with } f_{k} \in P^{k} .
$$

Let $1 \leq r<\infty$, and for every $1 \leq k \leq r$, let $N^{k} \subset P^{k}$ be a subspace such that

$$
\left.N^{k} \oplus \operatorname{im} \operatorname{ad}_{f_{0}}^{\Sigma}\right|_{P^{k}}=P^{k} .
$$

Then there exists an analytic diffeomorphism $\Phi$, sending an open neighborhood of 0 in $V^{N}$ to an open neighborhood of 0 in $V^{N}$, that conjugates the coupled cell network vector field $\gamma_{f}$ to a coupled cell network vector field $\gamma_{\bar{f}}$ with

$$
\bar{f}=f_{0}+\bar{f}_{1}+\bar{f}_{2}+\ldots \text { and } \bar{f}_{k} \in N^{k} \text { for all } 1 \leq k \leq r .
$$

Proof. [Sketch] We only sketch a proof without estimates here, because the construction of a normal form by means of "Lie transformations" is very well known.

For $g \in C^{\infty}\left(V^{n}, V\right)$ with $g(0)=0$, the time- $t$ flow $e^{t \gamma_{g}}$ defines a diffeomorphism of some open neighborhood of 0 in $V^{N}$ to another open neighborhood of 0 in $V^{N}$. Thus we can consider, for any $f \in C^{\infty}\left(V^{n}, V\right)$, the curve $t \mapsto\left(e^{t \gamma_{g}}\right)_{*} \gamma_{f} \in$ $C^{\infty}\left(V^{N}, V^{N}\right)$ of pushforward vector fields. This curve satisfies the linear differential equation

$$
\frac{d}{d t}\left(e^{t \gamma_{g}}\right)_{*} \gamma_{f}=\left.\frac{d}{d h}\right|_{h=0}\left(e^{h \gamma_{g}}\right)_{*}\left(\left(e^{t \gamma_{g}}\right)_{*} \gamma_{f}\right)=\left[\gamma_{g},\left(e^{t \gamma_{g}}\right)_{*} \gamma_{f}\right]=\operatorname{ad}_{\gamma_{g}}\left(\left(e^{t \gamma_{g}}\right)_{*} \gamma_{f}\right)
$$

where the second equality holds by definition of the Lie bracket of vector fields (5.14) and we have used the conventional definition of $\operatorname{ad}_{\gamma_{g}}: C^{\infty}\left(V^{N}, V^{N}\right) \rightarrow$ $C^{\infty}\left(V^{N}, V^{N}\right)$ as

$$
\operatorname{ad}_{\gamma_{g}}\left(\gamma_{f}\right):=\left[\gamma_{g}, \gamma_{f}\right]
$$

Solving the linear differential equation (6.18) together with the initial condition $\left(e^{0 \gamma_{g}}\right)_{*} \gamma_{f}=\gamma_{f}$, we find that the time-1 flow of $\gamma_{g}$ transforms $\gamma_{f}$ into

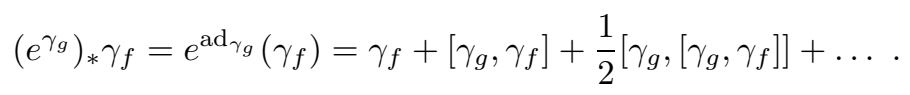

The main point of this proof is that by Theorem 5.1 the latter expression is also equal to

$$
\gamma_{f+[g, f]_{\Sigma}+\frac{1}{2}\left[g,[g, f]_{\Sigma}\right]_{\Sigma}+\ldots}=\gamma_{e^{\operatorname{ad}_{g}^{\Sigma}}(f)} .
$$

The diffeomorphism $\Phi$ in the statement of the theorem is now constructed as the composition of a sequence of time-1 flows $e^{\gamma_{g_{k}}}(1 \leq k \leq r)$ of coupled cell network vector fields $\gamma_{g_{k}}$ with $g_{k} \in P^{k}$. We first take $g_{1} \in P^{1}$, so that $\gamma_{f}$ is transformed by $e^{\gamma_{g_{1}}}$ into

$$
\left(e^{\gamma_{g_{1}}}\right)_{*} \gamma_{f}=\gamma_{e^{\operatorname{ad}_{g_{1}}^{\perp}}(f)}=\gamma_{f_{0}+f_{1}^{1}+f_{2}^{1}+\ldots}
$$


in which

$$
\begin{array}{ll}
f_{1}^{1}=f_{1}+\left[g_{1}, f_{0}\right]_{\Sigma} & \in P^{1} \\
f_{2}^{1}=f_{2}+\left[g_{1}, f_{1}\right]_{\Sigma}+\frac{1}{2}\left[g_{1},\left[g_{1}, f_{0}\right]_{\Sigma}\right]_{\Sigma} & \in P^{2} \\
f_{3}^{1}=f_{3}+\ldots & \in P^{3} \\
\text { etc. } &
\end{array}
$$

It is the fact that $\left.N^{1} \oplus \operatorname{im} \operatorname{ad}_{f_{0}}^{\Sigma}\right|_{P^{1}}=P^{1}$ that allows us to choose a (not necessarily unique) $g_{1} \in P^{1}$ in such a way that

$$
f_{1}^{1}=f_{1}+\left[g_{1}, f_{0}\right]_{\Sigma}=f_{1}-\operatorname{ad}_{f_{0}}^{\Sigma}\left(g_{1}\right) \in N^{1} .
$$

We proceed by choosing $g_{2} \in P^{2}$ in such a way that

$$
\left(e^{\gamma_{g_{2}}} \circ e^{\gamma_{g_{1}}}\right)_{*} \gamma_{f}=\left(e^{\gamma_{g_{2}}}\right)_{*}\left(\left(e^{\gamma_{g_{1}}}\right)_{*} \gamma_{f}\right)=\gamma_{f_{0}+f_{1}^{1}+f_{2}^{2}+\ldots}
$$

with $f_{2}^{2} \in N^{2}$. Continuing in this way, after $r$ steps we obtain that

$$
\Phi:=e^{\gamma_{g_{r}}} \circ \ldots \circ e^{\gamma_{g_{1}}}
$$

transforms $\gamma_{f}$ into $\Phi_{*} \gamma_{f}=\gamma_{\bar{f}}=\gamma_{f_{0}+\bar{f}_{1}+\ldots}$, where $\bar{f}_{k}=f_{k}^{k} \in N^{k}$ for all $1 \leq k \leq r$.

Being the composition of finitely many flows of polynomial coupled cell network vector fields, $\Phi$ is obviously analytic.

In applications, one is often interested in the bifurcations that occur in the dynamics of a map or differential equation under the variation of external parameters. In the case of coupled cell networks, we may for example assume that $f \in C^{\infty}\left(V^{n} \times \mathbb{R}^{p}, V\right)$ and let

$$
f^{\lambda}(X):=f(X ; \lambda)
$$

define a smooth parameter family in $C^{\infty}\left(V^{n}, V\right)$. Correspondingly, the coupled cell networks $\gamma_{f^{\lambda}}$ form a smooth parameter family in $C^{\infty}\left(V^{N}, V^{N}\right)$.

To formulate an appropriate normal form theorem for parameter families of coupled cell networks, we define for $k \geq-1$ and $l \geq 0$,

$$
P^{k, l}:=\left\{f: V^{n} \times \mathbb{R}^{p} \rightarrow V\right. \text { homogeneous polynomial }
$$

$$
\text { of degree } k+1 \text { in } X \text { and degree } l \text { in } \lambda \text { \}. }
$$

We observe that

$$
\left[P^{k, l}, P^{K, L}\right]_{\Sigma} \subset P^{k+K, l+L},
$$

which leads to the following

Theorem 6.2 (Coupled cell network normal form theorem with parameters). Let $\Sigma=\left\{\sigma_{1}, \ldots, \sigma_{n}\right\}$ be a semigroup, $f \in C^{\infty}\left(V^{n} \times \mathbb{R}^{p}, V\right)$ and $f(0 ; 0)=0$. We Taylor expand

$f=\left(f_{-1,1}+f_{-1,2}+\ldots\right)+\left(f_{0,0}+f_{0,1}+f_{0,2}+\ldots\right)+\left(f_{1,0}+f_{1,1}+f_{1,2}+\ldots\right)+\ldots$ with $f_{k, l} \in P^{k, l}$.

Let $1 \leq r_{1}, r_{2}<\infty$, and for every $-1 \leq k \leq r_{1}$ and $0 \leq l \leq r_{2}$, let $N^{k, l} \subset P^{k, l}$ be a subspace such that

$$
\left.N^{k, l} \oplus \operatorname{im} \operatorname{ad}_{f_{0,0}}^{\Sigma}\right|_{P^{k, l}}=P^{k, l} .
$$

Then there exists a polynomial family $\Phi^{\lambda}$ of analytic diffeomorphisms, defined for $\lambda$ in an open neighborhood of 0 in $\mathbb{R}^{p}$ and each sending an open neighborhood of 0 
in $V^{N}$ to an open neighborhood of 0 in $V^{N}$, with the property that $\Phi^{\lambda}$ conjugates $\gamma_{f^{\lambda}}$ to $\gamma_{\bar{f}^{\lambda}}$, where

$\bar{f}=\left(\bar{f}_{-1,1}+\bar{f}_{-1,2}+\ldots\right)+\left(f_{0,0}+\bar{f}_{0,1}+\bar{f}_{0,2}+\ldots\right)+\left(\bar{f}_{1,0}+\bar{f}_{1,1}+\bar{f}_{1,2}+\ldots\right)+\ldots$

and

$$
\bar{f}_{k, l} \in N^{k, l} \text { for all }-1 \leq k \leq r_{1} \text { and } 0 \leq l \leq r_{2} .
$$

Sketch of proof. The procedure of normalization is similar to the proof of Theorem 6.1. With respect to $\operatorname{ad}_{f_{0,0}}^{\Sigma}$, one consecutively normalizes

$$
\begin{gathered}
f_{1,0}, f_{2,0}, \ldots, f_{r_{1}, 0} ; f_{-1,1}, f_{0,1}, f_{1,1}, \ldots, f_{r_{1}, 1} ; \\
f_{-1,2}, f_{0,2}, f_{1,2}, \ldots, f_{r_{1}, 2} ; \ldots ; f_{-1, r_{2}}, f_{0, r_{2}}, f_{1, r_{2}}, \ldots, f_{r_{1}, r_{2}} .
\end{gathered}
$$

Because $\left[P^{k, l}, P^{K, L}\right]_{\Sigma} \subset P^{k+K, l+L}$, we see that once $f_{k, l}$ has been normalized to $\bar{f}_{k, l}$, it is not changed/affected anymore by any of the subsequent normalization transformations.

Of course, Theorem5.1implies that many other standard results from the theory of normal forms will have a counterpart in the context of semigroup coupled cell networks as well.

We will compute the normal forms of some network differential equations in Section 11

Example 6.3. Again, let $N=3$ and let $\sigma_{1}, \sigma_{2}, \sigma_{3}$ be defined as in Examples 2.3 and 2.4. If

$$
\gamma_{f}\left(x_{1}, x_{2}, x_{3}\right)=\left(f\left(x_{1}, x_{1}\right), f\left(x_{2}, x_{1}\right), f\left(x_{3}, x_{2}\right)\right)
$$

is a coupled cell network subject to $\left\{\sigma_{1}, \sigma_{2}\right\}$, then its normal form will in general be a network subject to $\left\{\sigma_{1}, \sigma_{2}, \sigma_{3}\right\}$, i.e.

$$
\overline{\gamma_{f}}\left(x_{1}, x_{2}, x_{3}\right)=\gamma_{\bar{f}}\left(x_{1}, x_{2}, x_{3}\right)=\left(\bar{f}\left(x_{1}, x_{1}, x_{1}\right), \bar{f}\left(x_{2}, x_{1}, x_{1}\right), \bar{f}\left(x_{3}, x_{2}, x_{1}\right)\right) .
$$

\section{SYMMETRY AND SYNCHRONY}

Symmetry [5], 6], [18, 25], 31] and synchrony [2], 3], 10, 11, 27, 32, [34, 38, [49, 51, 53. have obtained much attention in the literature on coupled cell networks. They generate and explain interesting patterns, including synchronized states [30, multirythms [19, 36], [44, [52, rotating waves [24, 32] and synchronized chaos 20, 24 and can lead to symmetry and synchrony breaking bifurcations; cf. [1, [4], [8], 99, [14, [15, [17, [22, [25], [31, [33], [43, [50]. In short, symmetry and synchrony heavily impact the dynamics and bifurcations of a network.

In this section, we relate some of the existing theory on symmetry and synchrony to the semigroup extension that we propose. More precisely, we show that the semigroup extension does not affect the symmetries or synchrony spaces of a network. This implies in particular that the symmetries and synchrony spaces of a network are also present in its normal form. The semigroup extension is thus quite harmless and very natural.

To start, let us say that a permutation $p:\{1, \ldots, N\} \rightarrow\{1, \ldots, N\}$ of the cells is a network symmetry for $\Sigma$ if it sends the inputs of a cell to the inputs of its image. That is, if

$$
p \circ \sigma_{j}=\sigma_{j} \circ p \text { for all } 1 \leq j \leq n .
$$


The permutations with this property obviously form a group. More importantly, they are of dynamical interest because the corresponding representations

$$
\lambda_{p}: V^{N} \rightarrow V^{N},\left(x_{1}, \ldots, x_{N}\right) \mapsto\left(x_{p(1)}, \ldots, x_{p(N)}\right)
$$

conjugate every coupled cell network map $\gamma_{f}$ to itself:

$$
\begin{aligned}
& \left.\left(\gamma_{f} \circ \lambda_{p}\right)_{i}(x)=f\left(\pi_{i}\left(x_{p(1)}, \ldots, x_{p(N)}\right)\right)=f\left(x_{p\left(\sigma_{1}(i)\right)}, \ldots, x_{p\left(\sigma_{n}(i)\right.}\right)\right) \\
& \left.=f\left(x_{\sigma_{1}(p(i))}, \ldots, x_{\sigma_{n}(p(i)}\right)\right)=f\left(\pi_{p(i)}(x)\right)=\left(\gamma_{f}(x)\right)_{p(i)}=\left(\lambda_{p} \circ \gamma_{f}\right)_{i}(x) .
\end{aligned}
$$

In turn, this means that when $t \mapsto\left(x_{1}(t), \ldots, x_{N}(t)\right)$ is a solution to the differential equations $\dot{x}=\gamma_{f}(x)$, then so is $t \mapsto\left(x_{p(1)}(t), \ldots, x_{p(N)}(t)\right)$. And similarly that when $m \mapsto\left(x_{1}^{(m)}, \ldots, x_{N}^{(m)}\right)$ is an orbit of the map $x^{(m+1)}=\gamma_{f}\left(x^{(m)}\right)$, then so is $m \mapsto\left(x_{p(1)}^{(m)}, \ldots, x_{p(N)}^{(m)}\right)$.

The following lemma states that network symmetries are trivially preserved by our semigroup extension:

Lemma 7.1. Let $\Sigma=\left\{\sigma_{1}, \ldots, \sigma_{n}\right\}$ be a collection of maps, not necessarily forming a semigroup, and $p:\{1, \ldots, N\} \rightarrow\{1, \ldots, N\}$ a permutation.

Then $p$ is a network symmetry for $\Sigma$ if and only if it is a network symmetry for the semigroup $\Sigma^{\prime}$ generated by $\Sigma$.

Proof. Elements of the semigroup $\Sigma^{\prime}$ are of the form $\sigma_{j_{1}} \circ \ldots \circ \sigma_{j_{l}}$ for certain $\sigma_{j_{k}} \in \Sigma$. But if $p \circ \sigma_{j_{k}}=\sigma_{j_{k}} \circ p$ for $k=1, \ldots, l$, then also $p \circ\left(\sigma_{j_{1}} \circ \ldots \circ \sigma_{j_{l}}\right)=\left(\sigma_{j_{1}} \circ \ldots \circ \sigma_{j_{l}}\right) \circ p$. Thus, the collection of network symmetries of $\Sigma$ is the same as the collection of network symmetries of $\Sigma^{\prime}$.

Lemma 7.1 implies in particular that the composition $\gamma_{f} \circ \gamma_{g}=\gamma_{f \circ \Sigma}$ and the Lie bracket $\left[\gamma_{f}, \gamma_{g}\right]=\gamma_{[f, g]_{\Sigma}}$ will exhibit the same network symmetries as $\gamma_{f}$ and $\gamma_{g}$.

Though not much more complicated, the situation is slightly more interesting for the synchronous solutions of a network. We recall that a synchrony space of a coupled cell network is an invariant subspace in which certain of the $x_{i}$ (with $1 \leq i \leq N)$ are equal. First of all, the following result is classical; see [24], [49].

Proposition 7.2. Let $\Sigma=\left\{\sigma_{1}, \ldots, \sigma_{n}\right\}$ be a collection of maps, not necessarily forming a semigroup, and $P=\left\{P_{1}, \ldots, P_{r}\right\}$ a partition of $\{1, \ldots, N\}$. The following are equivalent:

i) For all $1 \leq j \leq n$ and all $1 \leq k_{1} \leq r$ there exists $a 1 \leq k_{2} \leq r$ so that $\sigma_{j}\left(P_{k_{1}}\right) \subset P_{k_{2}}$.

ii) For every $f \in C^{\infty}\left(V^{n}, V\right)$ the subspace

$\operatorname{Syn}_{P}:=\left\{x \in V^{N} \mid x_{i_{1}}=x_{i_{2}}\right.$ when $i_{1}$ and $i_{2}$ are in the same element of $\left.P\right\}$ is an invariant submanifold for the dynamics of $\gamma_{f}$.

Proof. The subspace $\operatorname{Syn}_{P}$ is invariant under the flow of the differential equation $\dot{x}=\gamma_{f}(x)$ if and only if the vector field $\gamma_{f}$ is tangent to $\operatorname{Syn}_{P}$. Similarly, $\operatorname{Syn}_{P}$ is invariant under the map $x^{(m+1)}=\gamma_{f}\left(x^{(m)}\right)$ if and only if $\gamma_{f}$ sends $\operatorname{Syn}_{P}$ to itself. Both properties just mean that for all $x \in \operatorname{Syn}_{P}$ it holds that

$f\left(x_{\sigma_{1}\left(i_{1}\right)}, \ldots, x_{\sigma_{n}\left(i_{1}\right)}\right)=f\left(x_{\sigma_{1}\left(i_{2}\right)}, \ldots, x_{\sigma_{n}\left(i_{2}\right)}\right)$ for all $i_{1}, i_{2}$ in the same element of $P$. The latter statement holds for all $f \in C^{\infty}\left(V^{n}, V\right)$ if and only if for all $x \in \operatorname{Syn}_{P}$, $x_{\sigma_{j}\left(i_{1}\right)}=x_{\sigma_{j}\left(i_{2}\right)}$ for all $1 \leq j \leq n$ and all $i_{1}, i_{2}$ in the same element of $P$. 
It is not hard to see that this is true precisely when all $\sigma_{j} \in \Sigma$ map the elements of $P$ into elements of $P$.

A partition $P$ of $\{1, \ldots, N\}$ with property $i$ ) is sometimes called a balanced partition or balanced coloring and a subspace $\operatorname{Syn}_{P}$ satisfying property ii) a (robust) synchrony space.

The following result says that the synchrony spaces of a network do not change if one extends the network architecture to a semigroup:

Lemma 7.3. Let $\Sigma=\left\{\sigma_{1}, \ldots, \sigma_{n}\right\}$ be a collection of maps, not necessarily forming a semigroup, and $P=\left\{P_{1}, \ldots, P_{r}\right\}$ a partition of $\{1, \ldots, N\}$.

Then $\operatorname{Syn}_{P}$ is a (robust) synchrony space for $\Sigma$ if and only if it is a (robust) synchrony space for the semigroup $\Sigma^{\prime}$ generated by $\Sigma$.

Proof. Elements of the semigroup $\Sigma^{\prime}$ are of the form $\sigma_{j_{1}} \circ \ldots \circ \sigma_{j_{l}}$ for certain $\sigma_{j_{k}} \in \Sigma$. This implies that the elements of $\Sigma$ send the elements of $P$ inside elements of $P$ if and only if the elements of $\Sigma^{\prime}$ do. In other words: that the collection of balanced partitions of $\Sigma$ and of $\Sigma^{\prime}$ are the same. The result now follows from Proposition 7.2 .

Lemma 7.3 implies in particular that the composition $\gamma_{f} \circ \gamma_{g}=\gamma_{f \circ \Sigma}$ and the Lie bracket $\left[\gamma_{f}, \gamma_{g}\right]=\gamma_{[f, g]_{\Sigma}}$ will exhibit the same synchrony spaces as $\gamma_{f}$ and $\gamma_{g}$.

We conclude this section with the following simple but important observation:

Corollary 7.4. Let $\Sigma=\left\{\sigma_{1}, \ldots, \sigma_{n}\right\}$ be a collection of maps, not necessarily forming a semigroup, and $\gamma_{f}$ a coupled cell network vector field subject to $\Sigma$.

Then a local normal form $\gamma_{\bar{f}}$ of $\gamma_{f}$ has the same network symmetries and the same synchrony spaces as $\gamma_{f}$.

Proof. $\gamma_{\bar{f}}$ is a coupled cell network with respect to the semigroup $\Sigma^{\prime}$ generated by $\Sigma$. Thus, the result follows from Lemma 7.1 and Lemma 7.3 .

Example 7.5. Again, let $N=3$ and let $\sigma_{1}, \sigma_{2}, \sigma_{3}$ be defined as in Examples 2.3 and 2.4. Recall that a coupled cell network differential equation subject to $\left\{\sigma_{1}, \sigma_{2}\right\}$ is of the form

$$
\dot{x}_{1}=f\left(x_{1}, x_{1}\right), \dot{x}_{2}=f\left(x_{2}, x_{1}\right), \dot{x}_{3}=f\left(x_{3}, x_{2}\right) .
$$

These equations do not have any network symmetries, but they do admit the nontrivial balanced partitions

$$
\{1,2\} \cup\{3\} \text { and }\{1,2,3\} \text {. }
$$

The corresponding invariant synchrony spaces $\left\{x_{1}=x_{2}\right\}$ and $\left\{x_{1}=x_{2}=x_{3}\right\}$ are preserved in the normal form because the latter is a coupled cell network subject to $\left\{\sigma_{1}, \sigma_{2}, \sigma_{3}\right\}$.

\section{INPUT SYMMETRIES}

As mentioned in Remark 2.2 the admissible maps and vector fields of a network system can be determined from its groupoid of input equivalences; see [35, 38, and [51]. This groupoid consists of all possible ways to identify the inputs of cell $i$ with the inputs of cell $j$, for all the cells $1 \leq i, j \leq N$.

In case the network is homogeneous, the groupoid of the network is the obvious one that identifies the $k$-th input of cell $i$ with the $k$-th input of cell $j$ for all 
$1 \leq k \leq n$. No inputs can therefore be interchanged and, putting it equivalently, there exists only one way to identify the inputs of one cell among themselves. The so-called vertex group of the groupoid therefore consists of only one element.

On the other hand, many coupled cell networks that appear in the mathematical literature display a nontrivial network groupoid. This case arises when the response function $f$ is assumed invariant under the permutation of some of its inputs. Such an input symmetry can be dynamically relevant because it may give rise to nontrivial robust synchrony spaces.

In this section, we point out one condition under which an input symmetry can be preserved in the normal form of $f$. Although rather intuitive, this condition appears far from optimal. As a consequence, this section is not important for the remainder of this paper and can be skipped at first reading.

Concretely, an input symmetry is reflected by a permutation $q:\{1, \ldots, n\} \rightarrow$ $\{1, \ldots, n\}$ with the property that $f \circ \lambda_{q} \circ \pi_{i}=f \circ \pi_{i}$ for all $1 \leq i \leq N$. That is, for which

$$
f\left(x_{\sigma_{1}(i)}, \ldots, x_{\sigma_{n}(i)}\right)=f\left(x_{\sigma_{q(1)}(i)}, \ldots, x_{\sigma_{q(n)}(i)}\right) \text { for all } x \in V^{N} \text { and all } 1 \leq i \leq N .
$$

The input symmetries of $f$ obviously form a group. In fact, this group is equal to the vertex group of the network groupoid.

In the remainder of this section, we will only consider the case that an input symmetry gives rise to a network symmetry. This means that along with the permutation $q$ of the inputs $\{1, \ldots, n\}$ there exists a permutation $p$ of the cells $\{1, \ldots, N\}$ that sends the $j$-th input of each cell to the $q(j)$-th input of its image, i.e. that

$$
p \circ \sigma_{j}=\sigma_{q(j)} \circ p \text { for all } 1 \leq j \leq n .
$$

We call a permutation $q$ for which there exists a permutation $p$ so that (8.19) and (8.20) hold a dynamical input symmetry. We remark that if $p_{1} \circ \sigma_{j}=\sigma_{q_{1}(j)} \circ p_{1}$ and $p_{2} \circ \sigma_{j}=\sigma_{q_{2}(j)} \circ p_{2}$ for all $1 \leq j \leq n$, then

$$
\left(p_{1} \circ p_{2}\right) \circ \sigma_{j}=\sigma_{\left(q_{1} \circ q_{2}\right)(j)} \circ\left(p_{1} \circ p_{2}\right) .
$$

This implies that the dynamical input symmetries form a subgroup of the group of all input symmetries. They are precisely the input symmetries that correspond to a dynamical symmetry of the network:

Proposition 8.1. Let $p$ be a permutation of $\{1, \ldots, N\}$ and $q$ a permutation of $\{1, \ldots, n\}$. Assume that $p \circ \sigma_{j}=\sigma_{q(j)} \circ p$ for all $1 \leq j \leq n$ and that (8.19) holds. Then

$$
\gamma_{f} \circ \lambda_{p}=\lambda_{p} \circ \gamma_{f}
$$

Proof. First of all we claim that

when $p \circ \sigma_{j}=\sigma_{q(j)} \circ p$ for all $1 \leq j \leq n$, then $\pi_{i} \circ \lambda_{p}=\lambda_{q} \circ \pi_{p(i)}$ for all $1 \leq i \leq N$.

This follows from a little computation:

$$
\begin{gathered}
\left(\pi_{i} \circ \lambda_{p}\right)(x)=\pi_{i}\left(x_{p(1)}, \ldots, x_{p(N)}\right)=\left(x_{p\left(\sigma_{1}(i)\right)}, \ldots, x_{p\left(\sigma_{n}(i)\right)}\right) \\
=\left(x_{\sigma_{q(1)}(p(i))}, \ldots, x_{\sigma_{q(n)}(p(i))}\right)=\lambda_{q}\left(x_{\sigma_{1}(p(i))}, \ldots, x_{\sigma_{n}(p(i))}\right)=\left(\lambda_{q} \circ \pi_{p(i)}\right)(x) .
\end{gathered}
$$


Using (8.21) and our assumption (8.19) that $f \circ \lambda_{q}=f$ on every im $\pi_{i}$, we hence find that

$$
\left(\gamma_{f} \circ \lambda_{p}\right)_{i}=f \circ \pi_{i} \circ \lambda_{p}=f \circ \lambda_{q} \circ \pi_{p(i)}=f \circ \pi_{p(i)}=\left(\gamma_{f}\right)_{p(i)}=\left(\lambda_{p} \circ \gamma_{f}\right)_{i} .
$$

The relevance of dynamical input symmetries for normal forms is explained below. We first show that dynamical input symmetries survive the semigroup extension.

Lemma 8.2. Let $\Sigma=\left\{\sigma_{1}, \ldots, \sigma_{n}\right\}$ be a collection of maps, not necessarily forming a semigroup, and let $q:\{1, \ldots, n\} \rightarrow\{1, \ldots, n\}$ be a dynamical input symmetry for $\Sigma$.

Then the latter extends to a unique dynamical input symmetry

$$
q^{\prime}:\left\{1, \ldots, n, n+1, \ldots, n^{\prime}\right\} \rightarrow\left\{1, \ldots, n, n+1, \ldots, n^{\prime}\right\}
$$

for the semigroup $\Sigma^{\prime}=\left\{\sigma_{1}, \ldots, \sigma_{n}, \sigma_{n+1}, \ldots, \sigma_{n^{\prime}}\right\}$ generated by $\Sigma$.

Proof. Recall that elements of $\Sigma^{\prime}$ are of the form $\sigma_{j_{1}} \circ \ldots \circ \sigma_{j_{l}}$ for certain $\sigma_{j_{k}} \in \Sigma$. Assume now that $p \circ \sigma_{j_{1}}=\sigma_{q\left(j_{1}\right)} \circ p$ and $p \circ \sigma_{j_{2}}=\sigma_{q\left(j_{2}\right)} \circ p$. Then it follows that $p \circ \sigma_{\widetilde{\sigma}_{j_{1}}\left(j_{2}\right)}=p \circ\left(\sigma_{j_{1}} \circ \sigma_{j_{2}}\right)=\sigma_{q\left(j_{1}\right)} \circ p \circ \sigma_{j_{2}}=\left(\sigma_{q\left(j_{1}\right)} \circ \sigma_{q\left(j_{2}\right)}\right) \circ p=\sigma_{\widetilde{\sigma}_{q\left(j_{1}\right)}\left(q\left(j_{2}\right)\right)} \circ p$. This means that if an extension $q^{\prime}:\left\{1, \ldots, n, n+1, \ldots, n^{\prime}\right\} \rightarrow\{1, \ldots, n$, $\left.n+1, \ldots, n^{\prime}\right\}$ exists, then it must be unique and satisfy

$$
q^{\prime}\left(\widetilde{\sigma}_{j_{1}}\left(j_{2}\right)\right)=\widetilde{\sigma}_{q\left(j_{1}\right)}\left(q\left(j_{2}\right)\right) .
$$

If now $p \circ \sigma_{j_{3}}=\sigma_{q\left(j_{3}\right)} \circ p$ and $p \circ \sigma_{j_{4}}=\sigma_{q\left(j_{4}\right)} \circ p$ and $\widetilde{\sigma}_{j_{1}}\left(j_{2}\right)=\widetilde{\sigma}_{j_{3}}\left(j_{4}\right)$, then actually

$$
\sigma_{\widetilde{\sigma}_{q\left(j_{1}\right)}\left(q\left(j_{2}\right)\right)} \circ p=p \circ \sigma_{\widetilde{\sigma}_{j_{1}}\left(j_{2}\right)}=p \circ \sigma_{\widetilde{\sigma}_{j_{3}}\left(j_{4}\right)}=\sigma_{\widetilde{\sigma}_{q\left(j_{3}\right)}\left(q\left(j_{4}\right)\right)} \circ p .
$$

From this it follows that $\widetilde{\sigma}_{q\left(j_{1}\right)}\left(q\left(j_{2}\right)\right)=\widetilde{\sigma}_{q\left(j_{3}\right)}\left(q\left(j_{4}\right)\right)$ if $\widetilde{\sigma}_{j_{1}}\left(j_{2}\right)=\widetilde{\sigma}_{j_{3}}\left(j_{4}\right)$ and hence that $q^{\prime}$ is well defined.

The following result explains that network symmetries are preserved under taking compositions and Lie brackets:

Theorem 8.3. Let $\Sigma$ be a semigroup and assume $p \circ \sigma_{j}=\sigma_{q(j)} \circ p$ for all $1 \leq j \leq n$. Then

$$
\begin{aligned}
\left(f \circ \circ_{\Sigma} g\right) \circ \lambda_{q} & =\left(f \circ \lambda_{q}\right) \circ_{\Sigma}\left(g \circ \lambda_{q}\right) & & \text { on every } \operatorname{im} \pi_{i}, \\
{[f, g]_{\Sigma} \circ \lambda_{q} } & =\left[f \circ \lambda_{q}, g \circ \lambda_{q}\right]_{\Sigma} & & \text { on every } \operatorname{im} \pi_{i} .
\end{aligned}
$$

Proof. With a slight abuse of notation, we write

$$
\begin{aligned}
& (f \circ \Sigma g) \circ \lambda_{q} \circ \pi_{p(i)}=f\left(\ldots, g\left(A_{\sigma_{j}} \circ \lambda_{q} \circ \pi_{p(i)}\right), \ldots\right)=f\left(\ldots, g\left(A_{\sigma_{j}} \circ \pi_{i} \circ \lambda_{p}\right), \ldots\right) \\
& =f\left(\ldots, g\left(\pi_{\sigma_{j}(i)} \circ \lambda_{p}\right), \ldots\right)=f\left(\ldots, g\left(\lambda_{q} \circ \pi_{p\left(\sigma_{j}(i)\right)}\right), \ldots\right) \\
& =f\left(\ldots,\left(g \circ \lambda_{q}\right)\left(\pi_{p\left(\sigma_{j}(i)\right)}\right), \ldots\right)=f\left(\ldots,\left(g \circ \lambda_{q}\right)\left(\pi_{\sigma_{q(j)}(p(i))}\right), \ldots\right) \\
& =\left(f \circ \lambda_{q}\right)\left(\ldots,\left(g \circ \lambda_{q}\right)\left(\pi_{\sigma_{j}(p(i))}\right), \ldots\right)=\left(f \circ \lambda_{q}\right)\left(\ldots,\left(g \circ \lambda_{q}\right)\left(A_{\sigma_{j}} \circ \pi_{p(i)}\right), \ldots\right) \\
& =\left(\left(f \circ \lambda_{q}\right) \circ_{\Sigma}\left(g \circ \lambda_{q}\right)\right) \circ \pi_{p(i)} .
\end{aligned}
$$

This proves that $\left(f \circ_{\Sigma} g\right) \circ \lambda_{q}=\left(f \circ \lambda_{q}\right) \circ_{\Sigma}\left(g \circ \lambda_{q}\right)$ on each $\operatorname{im} \pi_{p(i)}$ and hence, because $p$ is invertible, on each $\operatorname{im} \pi_{i}$. The proof for the Lie bracket is similar. 
Finally, we conclude

Corollary 8.4. Let $\Sigma=\left\{\sigma_{1}, \ldots, \sigma_{n}\right\}$ be a collection of maps, not necessarily forming a semigroup, and $\gamma_{f}$ a coupled cell network vector field subject to $\Sigma$.

Then the local normal form $\gamma_{\bar{f}}$ of $\gamma_{f}$ can be chosen to have the same dynamical input symmetries as $\gamma_{f}$.

Proof. Let $G$ denote the group of dynamical input symmetries of $f=f_{0}+f_{1}+f_{2}+\ldots$ and let us define the set of $G$-invariant functions

$C_{G}^{\infty}\left(V^{n}, V\right):=\left\{g \in C^{\infty}\left(V^{n}, V\right) \mid g \circ \lambda_{q} \circ \pi_{i}=g \circ \pi_{i}\right.$ for all $q \in G$ and all $\left.1 \leq i \leq N\right\}$.

Theorem 8.3 implies that if $g, h \in C_{G}^{\infty}\left(V^{n}, V\right)$, then also

$$
[g, h]_{\Sigma} \in C_{G}^{\infty}\left(V^{n}, V\right) .
$$

The fact that $f \in C_{G}^{\infty}\left(V^{n}, V\right)$ moreover implies that

$$
f_{k} \in P_{G}^{k}:=\left\{g_{k} \in P^{k} \mid g_{k} \circ \lambda_{q} \circ \pi_{i}=g_{k} \circ \pi_{i} \text { for all } q \in G \text { and all } 1 \leq i \leq N\right\}
$$

is a $G$-invariant polynomial of degree $k+1$.

It clearly holds that $\left[P_{G}^{k}, P_{G}^{l}\right]_{\Sigma} \subset P_{G}^{k+l}$. As a consequence, we can repeat the proof of Theorem 6.1 by replacing $P^{k}$ with $P_{G}^{k}$ and choosing the normal form spaces $N_{G}^{k} \subset P_{G}^{k}$ so that

$$
\left.\operatorname{im} \operatorname{ad}_{f_{0}}\right|_{P_{G}^{k}} \oplus N_{G}^{k}=P_{G}^{k} .
$$

This produces a normal form $\bar{f} \in C_{G}^{\infty}\left(V^{n}, V\right)$.

Example 8.5. Consider the class of differential equations of the form

$$
\begin{aligned}
& \dot{x}_{1}=f\left(x_{1}, x_{2}, x_{2}, x_{1}\right), \\
& \dot{x}_{2}=f\left(x_{1}, x_{2}, x_{1}, x_{2}\right) .
\end{aligned}
$$

These differential equations have a semigroup coupled cell network structure with $N=2$ and $n=4 ;$ see Figure 3 .
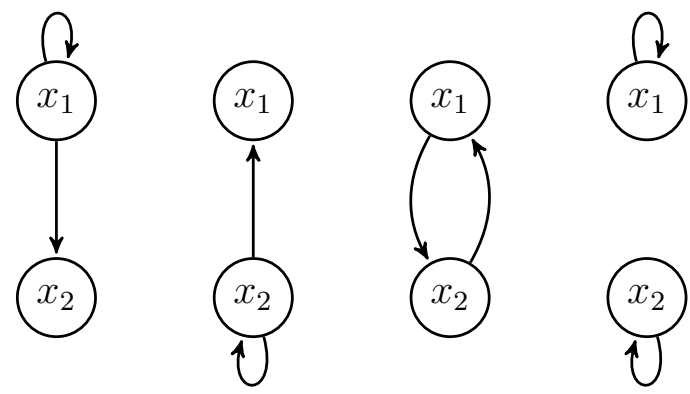

FIgURE 3 . The network with $N=2$ and $n=4$.

The semigroup $\Sigma$ in this case is the full non-Abelian semigroup of maps on 2 symbols. In other words, $\Sigma=\left\{\sigma_{1}, \sigma_{2}, \sigma_{3}, \sigma_{4}\right\}$, where

$$
\sigma_{1}(1)=1, \sigma_{1}(2)=1, \sigma_{2}(1)=2, \sigma_{2}(2)=2, \sigma_{3}(1)=2, \sigma_{3}(2)=1, \sigma_{4}(1)=1, \sigma_{4}(2)=2 \text {. }
$$

There is only one nontrivial permutation $p:\{1,2\} \rightarrow\{1,2\}$ of the cells, which is defined by $p(1):=2$ and $p(2):=1$. It is easily checked that

$$
p \circ \sigma_{1}=\sigma_{2} \circ p, p \circ \sigma_{2}=\sigma_{1} \circ p, p \circ \sigma_{3}=\sigma_{3} \circ p, p \circ \sigma_{4}=\sigma_{4} \circ p .
$$


In other words, $p \circ \sigma_{j}=\sigma_{q(j)} \circ p$ if we let $q:\{1,2,3,4\} \rightarrow\{1,2,3,4\}$ be defined by

$$
q(1)=2, q(2)=1, q(3)=3, q(4)=4 .
$$

Thus, the (in this case identical) invariances

$$
f\left(x_{1}, x_{2}, x_{2}, x_{1}\right)=f\left(x_{2}, x_{1}, x_{2}, x_{1}\right), f\left(x_{1}, x_{2}, x_{1}, x_{2}\right)=f\left(x_{2}, x_{1}, x_{1}, x_{2}\right)
$$

can be preserved in the normal form of $f$. These are precisely the invariances that make $\lambda_{p}:\left(x_{1}, x_{2}\right) \mapsto\left(x_{2}, x_{1}\right)$ a symmetry of the differential equations.

\section{SN-DECOMPOSITION}

We recall from the previous sections that when $f_{0} \in L\left(V^{n}, V\right)$, then $\operatorname{ad}_{f_{0}}^{\Sigma}: P^{k} \rightarrow$ $P^{k}$. The operators $\left.\operatorname{ad}_{f_{0}}^{\Sigma}\right|_{P^{k}}$ are called "homological operators", and they play an important role in normal form theory. This is first of all because the normal form spaces $N^{k} \subset P^{k}$ of Theorem 6.1 must be chosen complementary to their images, and secondly because in computing a normal form one needs to "invert" them when solving the homological equations $\operatorname{ad}_{f_{0}}^{\Sigma}\left(g_{k}\right)-h_{k} \in N^{k}$; see the proof of Theorem 6.1 .

For this reason, it is convenient to have at one's disposal the "SN-decompositions" (also called "Jordan-Chevalley decompositions") of the homological operators [7, [42], 47]. We recall that, since $P^{k}$ is finite-dimensional, the map $\left.\operatorname{ad}_{f_{0}}^{\Sigma}\right|_{P^{k}}$ admits a unique SN-decomposition

$$
\left.\operatorname{ad}_{f_{0}}^{\Sigma}\right|_{P^{k}}=\left(\left.\operatorname{ad}_{f_{0}}^{\Sigma}\right|_{P^{k}}\right)^{S}+\left(\left.\operatorname{ad}_{f_{0}}^{\Sigma}\right|_{P^{k}}\right)^{N}
$$

in which the map $\left(\left.\operatorname{ad}_{f_{0}}^{\Sigma}\right|_{P^{k}}\right)^{S}$ is semisimple, the map $\left(\left.\operatorname{ad}_{f_{0}}^{\Sigma}\right|_{P^{k}}\right)^{N}$ is nilpotent and the two maps $\left(\left.\operatorname{ad}_{f_{0}}^{\Sigma}\right|_{P^{k}}\right)^{S}$ and $\left(\left.\operatorname{ad}_{f_{0}}^{\Sigma}\right|_{P^{k}}\right)^{N}$ commute. The aim of this somewhat technical section is to characterize this $\mathrm{SN}$-decomposition in as simple a way as possible. We will do this in a number of steps, starting from the following technical result:

Proposition 9.1. Assume that $\Sigma$ is a semigroup. Then the linear map

$$
\left.\gamma\right|_{L\left(V^{n}, V\right)}: L\left(V^{n}, V\right) \rightarrow L\left(V^{N}, V^{N}\right) \text { sending } f_{0} \text { to } \gamma_{f_{0}} \text { is injective . }
$$

Proof. The definition $\left(\gamma_{f_{0}}\right)_{i}:=f_{0} \circ \pi_{i}$ implies that when $f_{0}$ is linear, then $\gamma_{f_{0}}=0$ precisely when $f_{0}$ vanishes on $\operatorname{im} \pi_{1}+\ldots+\operatorname{im} \pi_{N}$. Thus, all we need to show is that

$$
\operatorname{im} \pi_{1}+\ldots+\operatorname{im} \pi_{N}=V^{n} .
$$

Equivalently, we will show by contradiction that the map

$$
" \pi_{1}+\ldots+\pi_{N} ":\left(V^{N}\right)^{N} \rightarrow V^{n},\left(x^{(1)}, \ldots, x^{(N)}\right) \mapsto \pi_{1}\left(x^{(1)}\right)+\ldots+\pi_{N}\left(x^{(N)}\right)
$$

must be surjective. To this end, let us define for $1 \leq j \leq n$ the maps

$$
\begin{aligned}
\pi^{j}:\left(V^{N}\right)^{N} \rightarrow V \text { by } \pi^{j}\left(x^{(1)}, \ldots, x^{(N)}\right) & =\left(\pi_{1}\left(x^{(1)}\right)+\ldots+\pi_{N}\left(x^{(N)}\right)\right)_{j} \\
& =x_{\sigma_{j}(1)}^{(1)}+\ldots+x_{\sigma_{j}(N)}^{(N)} .
\end{aligned}
$$

In other words, $\pi^{j}$ is " $\pi_{1}+\ldots+\pi_{N}$ " followed by the projection to the $j$-th factor of $V^{n}$. In particular, if " $\pi_{1}+\ldots+\pi_{N}$ " is not surjective, then there is a relation of the form

$$
\pi^{j}=\sum_{k \neq j}^{n} \lambda_{k} \pi^{k} .
$$


This means that

$x_{\sigma_{j}(1)}^{(1)}+\ldots+x_{\sigma_{j}(N)}^{(N)}=\sum_{k \neq j} \lambda_{k}\left(x_{\sigma_{k}(1)}^{(1)}+\ldots+x_{\sigma_{k}(N)}^{(N)}\right)$ for all $x^{(1)}, \ldots, x^{(N)} \in V^{N}$.

It is clear that this can only be true if $\sigma_{j}=\sigma_{k}$ for some $k \neq j$. This is a contradiction, because we assumed that the elements of $\Sigma$ are distinct.

Next, we recall that when $f_{0} \in L\left(V^{n}, V\right)$, then $\gamma_{f_{0}} \in L\left(V^{N}, V^{N}\right)$, and thus also the latter has an SN-decomposition

$$
\gamma_{f_{0}}=\gamma_{f_{0}}^{S}+\gamma_{f_{0}}^{N}
$$

for certain $\gamma_{f_{0}}^{S}, \gamma_{f_{0}}^{N} \in L\left(V^{N}, V^{N}\right)$. We are going to relate the SN-decomposition of $\gamma_{f_{0}}$ to that of $\left.\operatorname{ad}_{f_{0}}^{\Sigma}\right|_{P^{k}}$. But first we show that both $\gamma_{f_{0}}^{S}$ and $\gamma_{f_{0}}^{N}$ are coupled cell network maps:

Lemma 9.2. For every $f_{0} \in L\left(V^{n}, V\right)$ there exist unique $f_{0}^{S}, f_{0}^{N} \in L\left(V^{n}, V\right)$ so that

$$
\gamma_{f_{0}}^{S}=\gamma_{f_{0}^{S}} \text { and } \gamma_{f_{0}}^{N}=\gamma_{f_{0}^{N}}
$$

It holds that $f_{0}=f_{0}^{S}+f_{0}^{N}$ and that $\left[f_{0}^{S}, f_{0}^{N}\right]_{\Sigma}=0$. In particular, $\operatorname{ad}_{f_{0}^{S}}^{\Sigma} \circ \operatorname{ad}_{f_{0}^{N}}^{\Sigma}=$ $\operatorname{ad}_{f_{0}^{N}}^{\Sigma} \circ \operatorname{ad}_{f_{0}^{S}}^{\Sigma}$.

Proof. We recall - see for instance [39, p. 17 - that both the semisimple part $\gamma_{f_{0}}^{S}$ and the nilpotent part $\gamma_{f_{0}}^{N}$ of $\gamma_{f_{0}}$ are polynomial functions of $\gamma_{f_{0}}$. More precisely, $\gamma_{f_{0}}^{S}=p\left(\gamma_{f_{0}}\right)$ and $\gamma_{f_{0}}^{N}=\gamma_{f_{0}}-p\left(\gamma_{f_{0}}\right)$, where

$$
p(\gamma)=a_{0} I+a_{1} \gamma+\ldots+a_{d} \gamma^{d}
$$

is a polynomial with coefficients $a_{0}, \ldots, a_{d} \in \mathbb{C}$.

Theorem 4.2 then implies that $\gamma_{f_{0}}^{S}=p\left(\gamma_{f_{0}}\right)=\gamma_{p\left(f_{0}\right)}=\gamma_{f_{0}^{S}}$ for $f_{0}^{S} \in L\left(V^{n}, V\right)$ defined as

$$
f_{0}^{S}=p\left(f_{0}\right)=a_{0} f_{0}+a_{1}\left(f_{0} \circ_{\Sigma} f_{0}\right)+\ldots+a_{d}\left(f_{0} \circ_{\Sigma} \ldots \circ_{\Sigma} f_{0}\right) .
$$

By Lemma 4.3 this $f_{0}^{S}$ is well defined, while by Proposition 9.1 it is unique. Similarly, $\gamma_{f_{0}}^{N}=\gamma_{f_{0}^{N}}$ for a well-defined and unique $f_{0}^{N}=f_{0}-p\left(f_{0}\right) \in L\left(V^{n}, V\right)$. Clearly, $f_{0}=f_{0}^{S}+f_{0}^{N}$.

Because $\left[f_{0}^{S}, f_{0}^{N}\right]_{\Sigma} \in L\left(V^{n}, V\right)$ and

$$
\gamma_{\left[f_{0}^{S}, f_{0}^{N}\right]_{\Sigma}}=\left[\gamma_{f_{0}^{S}}, \gamma_{f_{0}^{N}}\right]=\left[\gamma_{f_{0}}^{S}, \gamma_{f_{0}}^{N}\right]=0
$$

it follows from Proposition 9.1 that $\left[f_{0}^{S}, f_{0}^{N}\right]_{\Sigma}=0$. The Jacobi identity

$$
\left[\operatorname{ad}_{f_{0}^{S}}^{\Sigma}, \operatorname{ad}_{f_{0}^{N}}^{\Sigma}\right]=\operatorname{ad}_{\left[f_{0}^{S}, f_{0}^{N}\right]_{\Sigma}}^{\Sigma}
$$

then confirms that $\operatorname{ad}_{f_{0}^{S}}^{\Sigma}$ and $\operatorname{ad}_{f_{0}^{N}}^{\Sigma}$ commute as operators on $C^{\infty}\left(V^{n}, V\right)$.

Before we come to the desired characterization of the SN-decomposition of $\left.\operatorname{ad}_{f_{0}}^{\Sigma}\right|_{P^{k}}$, we need to make one simple observation. It concerns the fact that two functions $f, g \in C^{\infty}\left(V^{n}, V\right)$ generate the same network map (in the sense that $\left.\gamma_{f}=\gamma_{g}\right)$ if and only if $f-g \in \operatorname{ker} \gamma=\left\{h \mid \gamma_{h}=0\right\} \subset C^{\infty}\left(V^{n}, V\right)$. Thus, $\operatorname{ker} \gamma$ consists of those functions $h: V^{n} \rightarrow V$ that are irrelevant for the dynamics of coupled cell networks.

One can remark that when $f, g \in C^{\infty}\left(V^{n}, V\right)$ and $f \in \operatorname{ker} \gamma$, then $\gamma_{[f, g]_{\Sigma}}=$ $\left[\gamma_{f}, \gamma_{g}\right]=0$ and hence also $[f, g]_{\Sigma} \in \operatorname{ker} \gamma$. This means $\operatorname{ker} \gamma \subset C^{\infty}\left(V^{n}, V\right)$ is a Lie 
algebra ideal. In particular, it holds for every $f \in C^{\infty}\left(V^{n}, V\right)$ that the adjoint map $\operatorname{ad}_{f}^{\Sigma}: C^{\infty}\left(V^{n}, V\right) \rightarrow C^{\infty}\left(V^{n}, V\right)$ sends ker $\gamma$ to ker $\gamma$ and hence that $\operatorname{ad}_{f}^{\Sigma}$ descends to a well-defined map on $C^{\infty}\left(V^{n}, V\right) / \operatorname{ker} \gamma$. We can now formulate our result:

Theorem 9.3. For every $k=0,1,2, \ldots$ the maps

$$
\left(\left.\operatorname{ad}_{f_{0}}^{\Sigma}\right|_{P^{k}}\right)^{S} \text { and }\left.\operatorname{ad}_{f_{0}^{S}}^{\Sigma}\right|_{P^{k}} \text { respectively }\left(\left.\operatorname{ad}_{f_{0}}^{\Sigma}\right|_{P^{k}}\right)^{N} \text { and }\left.\operatorname{ad}_{f_{0}^{N}}^{\Sigma}\right|_{P^{k}}
$$

descend to the same map on $P^{k} / \operatorname{ker} \gamma$.

Proof. We start by repeating that $\operatorname{ad}_{f_{0}}^{\Sigma}$ maps ker $\gamma$ into itself and hence descends to a well-defined map on $C^{\infty}\left(V^{n}, V\right) / \operatorname{ker} \gamma$ that moreover sends $P^{k} / \operatorname{ker} \gamma$ into itself. More interestingly, since $\left(\left.\operatorname{ad}_{f_{0}}^{\Sigma}\right|_{P^{k}}\right)^{S}$ and $\left(\left.\operatorname{ad}_{f_{0}}^{\Sigma}\right|_{P^{k}}\right)^{N}$ are polynomial functions of $\left.\operatorname{ad}_{f_{0}}^{\Sigma}\right|_{P^{k}}$, also these latter maps send ker $\gamma$ into itself and thus descend to $P^{k} / \operatorname{ker} \gamma$.

For the actual proof of the theorem, we define for $k \geq 0$ the vector spaces

$$
\mathcal{P}^{k}:=\left\{\text { homogeneous polynomial vector fields on } V^{N} \text { of degree } k+1\right\} .
$$

It is clear that $\gamma: P^{k} / \operatorname{ker} \gamma \rightarrow \mathcal{P}^{k}$ is an injective linear map. Moreover, the computation

$$
\left(\gamma \circ \operatorname{ad}_{f_{0}}^{\Sigma}\right)\left(g_{k}\right)=\gamma_{\left[f_{0}, g_{k}\right]_{\Sigma}}=\left[\gamma_{f_{0}}, \gamma_{g_{k}}\right]=\left(\operatorname{ad}_{\gamma_{f_{0}}} \circ \gamma\right)\left(g_{k}\right)
$$

reveals that the maps $\operatorname{ad}_{f_{0}}^{\Sigma}: P^{k} / \operatorname{ker} \gamma \rightarrow P^{k} / \operatorname{ker} \gamma$ and $\operatorname{ad}_{\gamma_{f_{0}}}: \mathcal{P}^{k} \rightarrow \mathcal{P}^{k}$ are conjugate by the map $\gamma: P^{k} / \operatorname{ker} \gamma \rightarrow \mathcal{P}^{k}$. Similarly, $\operatorname{ad}_{f_{0}^{S}}^{\Sigma}$ is conjugate to $\operatorname{ad}_{\gamma_{f_{0}^{S}}}$ and $\operatorname{ad}_{f_{0}^{N}}^{\Sigma}$ is conjugate to $\operatorname{ad}_{\gamma_{f_{0}^{N}}}$. Now we recall the well-known fact that the SNdecomposition of $\left.\operatorname{ad}_{\gamma_{f_{0}}}\right|_{\mathcal{P}^{k}}$ is

$$
\begin{aligned}
\left.\operatorname{ad}_{\gamma_{f_{0}} \mid}\right|_{\mathcal{P}^{k}} & =\left(\left.\operatorname{ad}_{\gamma_{f_{0}}}\right|_{\mathcal{P}^{k}}\right)^{S}+\left(\left.\operatorname{ad}_{\gamma_{f_{0}}}\right|_{\mathcal{P}^{k}}\right)^{N}=\left.\operatorname{ad}_{\gamma_{f_{0}}^{S}}\right|_{\mathcal{P}^{k}}+\left.\operatorname{ad}_{\gamma_{f_{0}}^{N}}\right|_{\mathcal{P}^{k}} \\
& =\left.\operatorname{ad}_{\gamma_{f_{0}^{S}}}\right|_{\mathcal{P}^{k}}+\left.\operatorname{ad}_{\gamma_{f_{0}^{N}}}\right|_{\mathcal{P}^{k}} .
\end{aligned}
$$

Because $\gamma$ is injective, we have thus proved that

$$
\left.\operatorname{ad}_{f_{0}}^{\Sigma}\right|_{P^{k}}=\left.\operatorname{ad}_{f_{0}^{S}}^{\Sigma}\right|_{P^{k}}+\left.\operatorname{ad}_{f_{0}^{N}}^{\Sigma}\right|_{P^{k}}: P^{k} / \operatorname{ker} \gamma \rightarrow P^{k} / \operatorname{ker} \gamma
$$

is the SN-decomposition of the quotient map. Because the SN-decomposition of the quotient is the quotient of the $\mathrm{SN}$-decomposition, this proves the theorem.

Because the elements of ker $\gamma$ are dynamically completely irrelevant, for all practical purposes we can think of Theorem 9.3 as saying that

$$
\left." \operatorname{ad}_{f_{0}}^{\Sigma}\right|_{P^{k}}=\left.\operatorname{ad}_{f_{0}^{S}}^{\Sigma}\right|_{P^{k}}+\left.\operatorname{ad}_{f_{0}^{N}}^{\Sigma}\right|_{P^{k}} \text { is the SN-decomposition of }\left.\operatorname{ad}_{f_{0}}^{\Sigma}\right|_{P^{k}} " .
$$

This is very convenient, because it means that one can determine the SN-decompositions of all operators $\left.\operatorname{ad}_{f_{0}}^{\Sigma}\right|_{P^{k}}$ simultaneously by simply determining the splitting $f_{0}=f_{0}^{S}+f_{0}^{N}$.

Example 9.4. Even though by Proposition 9.1 the restriction $\left.\gamma\right|_{L\left(V^{n}, V\right)}$ is injective, the full map $\gamma: C^{\infty}\left(V^{n}, V\right) \rightarrow C^{\infty}\left(V^{N}, V^{N}\right)$ may fail to be so. This situation occurs when $\bigcup_{i=1}^{N} \operatorname{im} \pi_{i} \neq V^{n}$, because $\gamma_{f}=0$ already when $f$ vanishes on every $\operatorname{im} \pi_{i}$. This is the reason for the somewhat difficult formulation of Theorem 9.3 . 
To illustrate this phenomenon, we refer to Example 8.5 in which

$$
\pi_{1}\left(x_{1}, x_{2}\right)=\left(x_{1}, x_{2}, x_{2}, x_{1}\right) \text { and } \pi_{2}\left(x_{1}, x_{2}\right)=\left(x_{1}, x_{2}, x_{1}, x_{2}\right),
$$

so that in particular $\operatorname{im} \pi_{1} \cup \operatorname{im} \pi_{2} \neq V^{4}$. When for instance $V=\mathbb{R}$ in this example, then $\operatorname{ker} \gamma \subset C^{\infty}\left(\mathbb{R}^{4}, \mathbb{R}\right)$ is the ideal generated by

$\left(X_{1}-X_{4}\right)\left(X_{1}-X_{3}\right),\left(X_{1}-X_{4}\right)\left(X_{2}-X_{4}\right),\left(X_{2}-X_{3}\right)\left(X_{1}-X_{3}\right)$ and $\left(X_{2}-X_{3}\right)\left(X_{2}-X_{4}\right)$.

We conclude this section with the following dynamical implication of Theorem 9.3 .

Corollary 9.5. Let $0 \leq r<\infty$. Then it can be arranged that the normal form $\bar{f}=f_{0}+\bar{f}_{1}+\ldots \in C^{\infty}\left(V^{n}, V\right)$ of an $f=f_{0}+\bar{f}_{1}+\ldots \in C^{\infty}\left(V^{n}, V\right)$ has the special property that the truncated normal form coupled cell map/vector field $\gamma_{f_{0}+\bar{f}_{1}+\ldots+\bar{f}_{r}}$ commutes with the continuous family of maps

$$
t \mapsto e^{t \gamma_{f_{0}^{S}}}
$$

Proof. Recall that each one of the normal form spaces $N^{k} \subset P^{k}$ of Theorem 6.1 is required to have the property that $\left.N^{k} \oplus \operatorname{im} \operatorname{ad}_{f_{0}}^{\Sigma}\right|_{P^{k}}=P^{k}$. It is not hard to see that whenever

$$
N^{k} \subset \operatorname{ker}\left(\left.\operatorname{ad}_{f_{0}}^{\Sigma}\right|_{P^{k}}\right)^{S} \text { is complementary to } \operatorname{im}\left(\left.\operatorname{ad}_{f_{0}}^{\Sigma}\right|_{P^{k}}\right)^{N},
$$

then this condition is fulfilled. Thus, let us choose $N^{k}$ so that it satisfies (9.25). The fact that $\left(\left.\operatorname{ad}_{f_{0}^{S}}^{\Sigma}\right|_{P^{k}}\right)^{S}$ and $\left.\operatorname{ad}_{f_{0}^{S}}^{\Sigma}\right|_{P^{k}}$ descend to the same map on $P^{k} /$ ker $\gamma$ implies in particular that for such $N^{k}$ it holds that $\operatorname{ad}_{f_{0}^{S}}^{\Sigma}\left(N^{k}\right) \subset \operatorname{ker} \gamma$.

Now let $\bar{f}=f_{0}+\bar{f}_{1}+\bar{f}_{2}+\ldots$ be any normal form of $f$ of order $r$ with respect to the $N^{k}$, meaning that $\bar{f}_{k} \in N^{k}$ for all $1 \leq k \leq r$. Such a normal form exists by Theorem 6.1. Then it holds that $\operatorname{ad}_{f_{0}^{S}}^{\Sigma}\left(\bar{f}_{k}\right) \in \operatorname{ker} \gamma$, and in view of Theorem 4.2 we therefore have

$$
\left[\gamma_{f_{0}^{S}}, \gamma_{f_{0}+\bar{f}_{1} \ldots+\bar{f}_{r}}\right]=\gamma_{\left[f_{0}^{S}, f_{0}+\bar{f}_{1}+\ldots+\bar{f}_{r}\right]_{\Sigma}}=\gamma_{\mathrm{ad}_{f_{0}^{S}}^{\Sigma}\left(f_{0}+\bar{f}_{1}+\ldots+\bar{f}_{r}\right)}=0 .
$$

Hence, $\left.\frac{d}{d t}\right|_{t=0}\left(e^{t \gamma_{f_{0}^{S}}}\right)_{*}\left(\gamma_{f_{0}+\bar{f}_{1} \ldots+\bar{f}_{r}}\right)=0$, and thus the truncated normal form commutes with the flow $t \mapsto e^{t \gamma_{f_{0}^{S}}}$ of the coupled cell network vector field $\gamma_{f_{0}^{S}}$.

The continuous family

$$
t \mapsto e^{t \gamma_{f_{0}^{S}}}
$$

of transformations of $V^{N}$ is called a normal form symmetry. This symmetry is sometimes used to characterize vector fields that are in normal form. It also plays an important role in finding periodic solutions near equilibria of the vector field $\gamma_{f}$, using for example the method of Lyapunov-Schmidt reduction [12], 23], 28], 33], [37.

\section{A FUndAMENTAL SEMigroup NETWORK}

As a byproduct of Theorem 4.1. and perhaps as a curiosity, we will show in this section that the dynamics of $\gamma_{f}$ on $V^{N}$ is conjugate to the dynamics of a certain network $\Gamma_{f}$ on $V^{n}$. We will argue that $\Gamma_{f}$ acts as a "fundamental network" for $\gamma_{f}$. 
We recall that if $\Sigma=\left\{\sigma_{1}, \ldots, \sigma_{n}\right\}$ is a semigroup, then every $\sigma_{j} \in \Sigma$ induces a map

$$
\widetilde{\sigma}_{j}:\{1, \ldots, n\} \rightarrow\{1, \ldots, n\} \text { via the formula } \sigma_{\widetilde{\sigma}_{j}(k)}=\sigma_{k} \circ \sigma_{j} .
$$

We saw that $\widetilde{\sigma_{j_{1}} \circ \sigma_{j_{2}}}=\widetilde{\sigma}_{j_{1}} \circ \widetilde{\sigma}_{j_{2}}$, and hence the collection $\widetilde{\Sigma}:=\left\{\widetilde{\sigma}_{1}, \ldots, \widetilde{\sigma}_{n}\right\}$ is closed under composition. One can now study coupled cell networks subject to $\widetilde{\Sigma}$. They have the form

$\Gamma_{f}: V^{n} \rightarrow V^{n}$ with $\left(\Gamma_{f}\right)_{j}(X):=f\left(X_{\widetilde{\sigma}_{1}(j)}, \ldots, X_{\widetilde{\sigma}_{n}(j)}\right)=f\left(A_{\sigma_{j}} X\right)$ for all $1 \leq j \leq n$.

The following theorem demonstrates that $\gamma_{f}$ and $\Gamma_{f}$ are dynamically related:

Theorem 10.1. All maps $\pi_{i}: V^{N} \rightarrow V^{n}$ conjugate $\gamma_{f}$ to $\Gamma_{f}$; that is,

$$
\Gamma_{f} \circ \pi_{i}=\pi_{i} \circ \gamma_{f} \text { for all } 1 \leq i \leq N .
$$

Proof. For $x \in V^{N}$ we have that

$$
\left(\Gamma_{f} \circ \pi_{i}\right)_{j}(x)=f\left(\left(A_{\sigma_{j}} \circ \pi_{i}\right)(x)\right)=f\left(\pi_{\sigma_{j}(i)}(x)\right)=\left(\gamma_{f}(x)\right)_{\sigma_{j}(i)}=\left(\pi_{i} \circ \gamma_{f}\right)_{j}(x) .
$$

Theorem 10.1 implies that every $\pi_{i}$ sends integral curves of $\gamma_{f}$ to integral curves of $\Gamma_{f}$ and discrete-time orbits of $\gamma_{f}$ to discrete-time orbits of $\Gamma_{f}$.

In addition, the dynamics of $\gamma_{f}$ can be reconstructed from the dynamics of $\Gamma_{f}$. More precisely, when $X_{(i)}(t)$ are integral curves of $\Gamma_{f}$ with $X_{(i)}(0)=\pi_{i}(x(0))$, then an integral curve $x(t)$ of $\gamma_{f}$ can simply be obtained by integration of the equations

$$
\dot{x}_{i}(t)=f\left(X_{(i)}(t)\right) \text { for } 1 \leq i \leq N .
$$

Similarly, if $X_{(i)}^{(m)}$ are discrete-time orbits of $\Gamma_{f}$ with $X_{(i)}^{(m)}(0)=\pi_{i}(x(0))$, then $x_{i}^{(m+1)}:=f\left(X_{(i)}^{(m)}\right)$ defines a discrete-time orbit of $\gamma_{f}$.

The transition from $\gamma_{f}$ to $\Gamma_{f}$ is thus reminiscent of the symmetry reduction of an equivariant dynamical system: the dynamics of $\gamma_{f}$ descends to the dynamics of $\Gamma_{f}$, and the dynamics of $\gamma_{f}$ can be reconstructed from that of $\Gamma_{f}$ by means of integration. Nevertheless, $n$ can of course be both smaller and larger than $N$. In the latter case, the dynamics of $\Gamma_{f}$ may be much richer than that of $\gamma_{f}$, and it is confusing to speak of reduction. In either case, $\Gamma_{f}$ captures all the dynamics of $\gamma_{f}$.

Example 10.2. Again, let $N=3$ and let $\sigma_{1}, \sigma_{2}, \sigma_{3}$ be defined as in Example 2.4. Recall

$$
\begin{aligned}
& A_{\sigma_{1}}\left(X_{1}, X_{2}, X_{3}\right)=\left(X_{1}, X_{2}, X_{3}\right), \\
& A_{\sigma_{2}}\left(X_{1}, X_{2}, X_{3}\right)=\left(X_{2}, X_{3}, X_{3}\right), \\
& A_{\sigma_{3}}\left(X_{1}, X_{2}, X_{3}\right)=\left(X_{3}, X_{3}, X_{3}\right) .
\end{aligned}
$$

This means that the network map $\Gamma_{f}$ is given by

$$
\Gamma_{f}\left(X_{1}, X_{2}, X_{3}\right)=\left(f\left(X_{1}, X_{2}, X_{3}\right), f\left(X_{2}, X_{3}, X_{3}\right), f\left(X_{3}, X_{3}, X_{3}\right)\right) .
$$

In this example, the conjugacies from $\gamma_{f}$ to $\Gamma_{f}$ are

$$
\begin{aligned}
& \pi_{1}:\left(x_{1}, x_{2}, x_{3}\right) \mapsto\left(X_{1}, X_{2}, X_{3}\right):=\left(x_{1}, x_{1}, x_{1}\right), \\
& \pi_{2}:\left(x_{1}, x_{2}, x_{3}\right) \mapsto\left(X_{1}, X_{2}, X_{3}\right):=\left(x_{2}, x_{1}, x_{1}\right), \\
& \pi_{3}:\left(x_{1}, x_{2}, x_{3}\right) \mapsto\left(X_{1}, X_{2}, X_{3}\right):=\left(x_{3}, x_{2}, x_{1}\right) .
\end{aligned}
$$

The conjugacy $\pi_{3}$ is bijective, which explains that Figures 1 and 4 are isomorphic. 

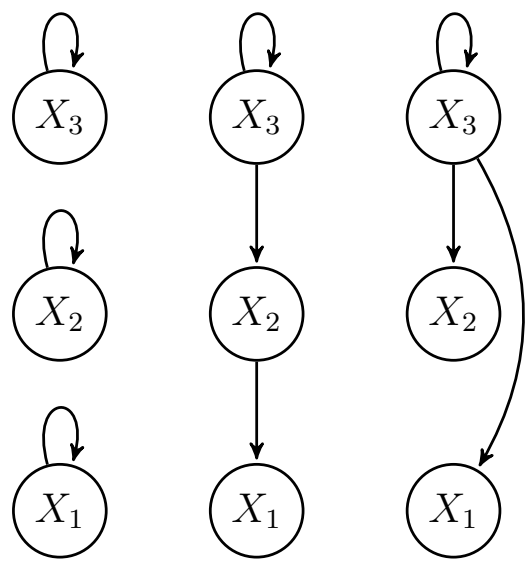

FiguRE 4. The fundamental network of our three cell feed-forward network.

Example 10.3. Recall Example 8.5 that features a semigroup with 4 elements. To determine the maps $A_{\sigma_{j}}$, we compute the multiplication table of this semigroup:

\begin{tabular}{c|cccc}
$\circ$ & $\sigma_{1}$ & $\sigma_{2}$ & $\sigma_{3}$ & $\sigma_{4}$ \\
\hline$\sigma_{1}$ & $\sigma_{1}$ & $\sigma_{1}$ & $\sigma_{1}$ & $\sigma_{1}$ \\
$\sigma_{2}$ & $\sigma_{2}$ & $\sigma_{2}$ & $\sigma_{2}$ & $\sigma_{2}$ \\
$\sigma_{3}$ & $\sigma_{2}$ & $\sigma_{1}$ & $\sigma_{4}$ & $\sigma_{3}$ \\
$\sigma_{4}$ & $\sigma_{1}$ & $\sigma_{2}$ & $\sigma_{3}$ & $\sigma_{4}$
\end{tabular}.

This implies that

$$
\begin{aligned}
& A_{\sigma_{1}}\left(X_{1}, X_{2}, X_{3}, X_{4}\right)=\left(X_{1}, X_{2}, X_{2}, X_{1}\right), \\
& A_{\sigma_{2}}\left(X_{1}, X_{2}, X_{3}, X_{4}\right)=\left(X_{1}, X_{2}, X_{1}, X_{2}\right), \\
& A_{\sigma_{3}}\left(X_{1}, X_{2}, X_{3}, X_{4}\right)=\left(X_{1}, X_{2}, X_{4}, X_{3}\right), \\
& A_{\sigma_{4}}\left(X_{1}, X_{2}, X_{3}, X_{4}\right)=\left(X_{1}, X_{2}, X_{3}, X_{4}\right) .
\end{aligned}
$$

Hence, the corresponding fundamental network is given by

$$
\begin{aligned}
& \dot{X}_{1}=f\left(X_{1}, X_{2}, X_{2}, X_{1}\right), \\
& \dot{X}_{2}=f\left(X_{1}, X_{2}, X_{1}, X_{2}\right), \\
& \dot{X}_{3}=f\left(X_{1}, X_{2}, X_{4}, X_{3}\right), \\
& \dot{X}_{4}=f\left(X_{1}, X_{2}, X_{3}, X_{4}\right) .
\end{aligned}
$$

This fundamental network has been depicted in Figure 5. It is clear that the maps

$$
\begin{aligned}
& \pi_{1}:\left(x_{1}, x_{2}\right) \mapsto\left(X_{1}, X_{2}, X_{3}, X_{4}\right):=\left(x_{1}, x_{2}, x_{2}, x_{1}\right), \\
& \pi_{2}:\left(x_{1}, x_{2}\right) \mapsto\left(X_{1}, X_{2}, X_{3}, X_{4}\right):=\left(x_{1}, x_{2}, x_{1}, x_{2}\right)
\end{aligned}
$$

conjugate $\gamma_{f}$ to $\Gamma_{f}$.

The advantage of studying $\Gamma_{f}$ instead of $\gamma_{f}$ is that the definition $\left(\Gamma_{f}\right)_{j}:=f \circ A_{\sigma_{j}}$ explicitly displays the representation of the semigroup $\Sigma$, whereas the definition 


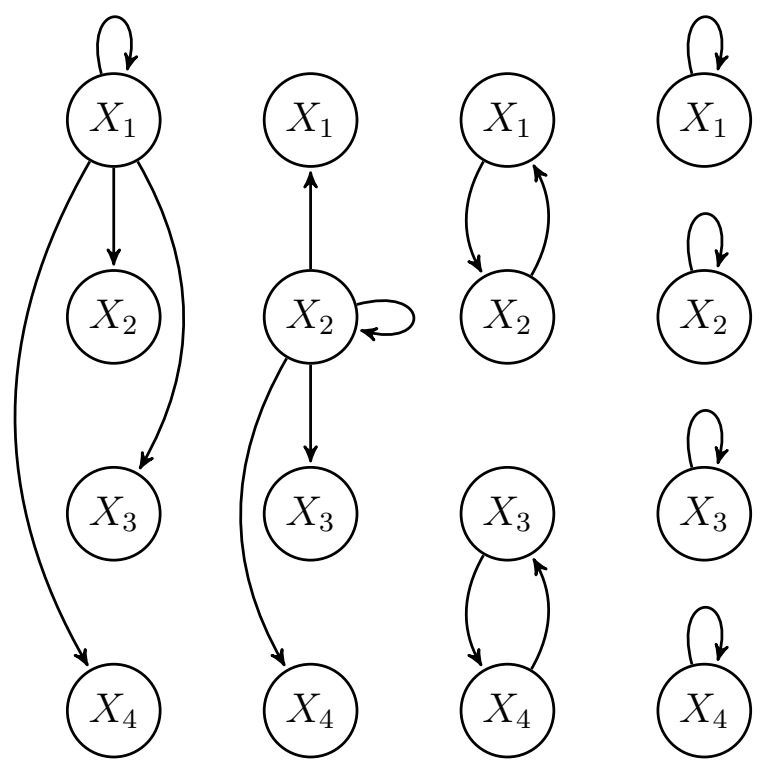

Figure 5. A fundamental network with $n=4$.

$\left(\gamma_{f}\right)_{i}:=f \circ \pi_{i}$ clearly does not. This has as a consequence that the transformation formulas for the composition and the Lie bracket become completely natural:

Lemma 10.4. It holds that

$$
\Gamma_{f} \circ \Gamma_{g}=\Gamma_{f \circ \Sigma g} \text { and }\left[\Gamma_{f}, \Gamma_{g}\right]=\Gamma_{[f, g]_{\Sigma}} .
$$

Proof. First of all,

$$
\begin{gathered}
A_{\sigma_{j}}\left(g\left(A_{\sigma_{1}} X\right), \ldots, g\left(A_{\sigma_{n}} X\right)\right)=\left(g\left(A_{\sigma_{\widetilde{\sigma}_{1}(j)}} X\right), \ldots, g\left(A_{\sigma_{\widetilde{\sigma}_{n}(j)}} X\right)\right) \\
=\left(g\left(A_{\sigma_{1} \circ \sigma_{j}} X\right), \ldots, g\left(A_{\sigma_{n} \circ \sigma_{j}} X\right)\right) .
\end{gathered}
$$

This gives that

$$
\begin{aligned}
\left(\Gamma_{f} \circ \Gamma_{g}\right)_{j}(X) & =f\left(A_{\sigma_{j}}\left(g\left(A_{\sigma_{1}} X\right), \ldots, g\left(A_{\sigma_{n}} X\right)\right)\right) \\
& =f\left(g\left(A_{\sigma_{1} \circ \sigma_{j}} X\right), \ldots, g\left(A_{\sigma_{n} \circ \sigma_{j}} X\right)\right) \\
& =f\left(g\left(A_{\sigma_{1}}\left(A_{\sigma_{j}} X\right)\right), \ldots, g\left(A_{\sigma_{n}}\left(A_{\sigma_{j}} X\right)\right)\right) \\
& =\left(f \circ \circ_{\Sigma} g\right)\left(A_{\sigma_{j}} X\right)=\left(\Gamma_{f \circ \Sigma} g\right)_{j}(X) .
\end{aligned}
$$

The computation for the Lie bracket is similar.

We stress that Lemma 10.4 holds due to the definition $\left(\Gamma_{f}\right)_{j}:=f \circ A_{\sigma_{j}}$ and the fact that $\sigma_{j} \mapsto A_{\sigma_{j}}$ is a homomorphism. Lemma 10.4 implies for example that the symbolic computation of the normal form of $\Gamma_{f}$ is the same as the symbolic computation of the normal form of $\gamma_{f}$.

We propose to call $\Gamma_{f}$ the fundamental network of $\gamma_{f}$. Two properties make this fundamental network fundamental: first of all, the network architecture of the fundamental network only depends on the multiplicative structure of the semigroup $\Sigma$ and not on the explicit realization of $\Sigma$ itself; in particular, it does not depend on $N$. This means that two semigroup networks have isomorphic fundamental 
networks if and only if their semigroups are isomorphic. The second fundamental property of the fundamental network is that it is equal to its own fundamental network, if the latter is defined. This follows from Proposition 10.5 below, in which we call a homomorphism of semigroups faithful if it is injective.

Proposition 10.5. Assume that the homomorphism $\sigma_{j} \mapsto \widetilde{\sigma}_{j}$ is faithful. Then

$$
\widetilde{\widetilde{\sigma}}_{j}=\widetilde{\sigma}_{j} \text {, and therefore } A_{\sigma_{j}}=A_{\widetilde{\sigma}_{j}} \text { for all } 1 \leq j \leq n .
$$

Proof. Recall that $\widetilde{\Sigma}=\left\{\widetilde{\sigma}_{1}, \ldots, \widetilde{\sigma}_{n}\right\}$ is closed under composition. Thus, the condition that the homomorphism $\sigma_{j} \mapsto \widetilde{\sigma}_{j}$ from $\Sigma$ to $\widetilde{\Sigma}$ is faithful just means that $\widetilde{\Sigma}$ is a semigroup. In particular, each map $\widetilde{\widetilde{\sigma}}_{j}:\{1, \ldots, n\} \rightarrow\{1, \ldots, n\}$ is then well defined. Now we compute

$$
\widetilde{\sigma}_{\widetilde{\sigma}_{j}(k)}=\widetilde{\sigma}_{j} \circ \widetilde{\sigma}_{k}=\widetilde{\sigma_{j} \circ \sigma_{k}}=\widetilde{\sigma_{\widetilde{\sigma}_{j}(k)}}=\widetilde{\sigma}_{\widetilde{\sigma}_{j}(k)} .
$$

This proves that $\widetilde{\widetilde{\sigma}}_{j}=\widetilde{\sigma}_{j}$ for all $1 \leq j \leq n$ and hence that $A_{\widetilde{\sigma}_{j}}=A_{\sigma_{j}}$ for all $1 \leq j \leq n$.

Proposition 10.5 brings up the question of when the homomorphism $\sigma_{j} \mapsto \widetilde{\sigma}_{j}$ is faithful, i.e. under which conditions the elements of $\Sigma$ all have different leftmultiplicative behavior. We give a partial answer to this question in Remark 10.9 below. The upshot of this remark is that one may essentially always assume the homomorphism to be faithful.

We finish this section with a few simple observations on synchrony and symmetry for $\Gamma_{f}$. First of all, a direct consequence of Theorem 10.1 is that each $\operatorname{im} \pi_{i} \subset V^{n}$ is an invariant subspace for the dynamics of $\Gamma_{f}$. Interestingly, another way to see this is by the following

Proposition 10.6. Every $\operatorname{im} \pi_{i} \subset V^{n}$ is a robust synchrony space for the $\Gamma_{f}$ 's.

Proof. Let us define a partition $P$ of $\{1, \ldots, n\}$ by letting $1 \leq j_{1}, j_{2} \leq n$ be in the same element of $P$ if and only if $\sigma_{j_{1}}(i)=\sigma_{j_{2}}(i)$. Then

$$
\begin{aligned}
\operatorname{Syn}_{P} & =\left\{X \in V^{n} \mid X_{j_{1}}=X_{j_{2}} \text { when } \sigma_{j_{1}}(i)=\sigma_{j_{2}}(i)\right\} \\
& =\left\{\left(x_{\sigma_{1}(i)}, \ldots, x_{\sigma_{n}(i)}\right) \mid x \in V^{N}\right\}=\operatorname{im} \pi_{i} .
\end{aligned}
$$

It remains to show that the partition $P$ is balanced for $\widetilde{\Sigma}$. This is easy though: when $1 \leq j_{1}, j_{2} \leq n$ are in the same element of $P$, then it holds for all $1 \leq k \leq n$ that

$$
\sigma_{\widetilde{\sigma}_{k}\left(j_{1}\right)}(i)=\left(\sigma_{k} \circ \sigma_{j_{1}}\right)(i)=\left(\sigma_{k} \circ \sigma_{j_{2}}\right)(i)=\sigma_{\widetilde{\sigma}_{k}\left(j_{2}\right)}(i),
$$

where the middle equality holds because $\sigma_{j_{1}}(i)=\sigma_{j_{2}}(i)$. This proves that also $\widetilde{\sigma}_{k}\left(j_{1}\right)$ and $\widetilde{\sigma}_{k}\left(j_{2}\right)$ are in the same element of $P$ and hence that the elements of $\widetilde{\Sigma}$ preserve $P$.

Recall that $A_{\sigma_{j}} \circ \pi_{i}=\pi_{\sigma_{j}(i)}$. This implies that $A_{\sigma_{j}}$ sends the $\Gamma_{f}$-invariant subspace $\operatorname{im} \pi_{i}$ to the $\Gamma_{f}$-invariant subspace $\operatorname{im} \pi_{\sigma_{j}(i)}$. But much more is true: the following result shows that $A_{\sigma_{j}}$ sends all orbits of $\Gamma_{f}$ to orbits of $\Gamma_{f}$, even though $A_{\sigma_{j}}$ may not be invertible.

\section{Proposition 10.7.}

$$
\Gamma_{f} \circ A_{\sigma_{j}}=A_{\sigma_{j}} \circ \Gamma_{f}
$$


Proof.

$$
\begin{aligned}
& \left(\Gamma_{f} \circ A_{\sigma_{j}}\right)_{k}(X)=f\left(A_{\sigma_{k}} \circ A_{\sigma_{j}} X\right)=f\left(A_{\sigma_{k} \circ \sigma_{j}} X\right) \\
& =f\left(A_{\sigma_{\widetilde{\sigma}_{k}(j)}} X\right)=\left(\Gamma_{f}\right)_{\widetilde{\sigma}_{k}(j)}(X)=\left(A_{\sigma_{j}} \circ \Gamma_{f}\right)_{k}(X) .
\end{aligned}
$$

The final result of this section shows that $\Gamma_{f}$ may have even more symmetry: the dynamical input symmetries of $\gamma_{f}$ are true symmetries of $\Gamma_{f}$.

Proposition 10.8. If $p$ is a permutation of $\{1, \ldots, N\}$ and $q$ is a permutation of $\{1, \ldots, n\}$ so that $p \circ \sigma_{j}=\sigma_{q(j)} \circ p$ for all $1 \leq j \leq n$ and if $f \circ \lambda_{q} \circ \pi_{i}=f \circ \pi_{i}$ for all $1 \leq i \leq N$, then

$$
\Gamma_{f} \circ \lambda_{q}=\lambda_{q} \circ \Gamma_{f} \text { on every } \operatorname{im} \pi_{i} .
$$

Proof. Recall that under the conditions of the proposition, it holds that $\pi_{i} \circ \lambda_{p}=$ $\lambda_{q} \circ \pi_{p(i)}$ and that from this it followed that $\lambda_{p} \circ \gamma_{f}=\gamma_{f} \circ \lambda_{p}$. As a consequence,

$\Gamma_{f} \circ \lambda_{q} \circ \pi_{p(i)}=\Gamma_{f} \circ \pi_{i} \circ \lambda_{p}=\pi_{i} \circ \gamma_{f} \circ \lambda_{p}=\pi_{i} \circ \lambda_{p} \circ \gamma_{f}=\lambda_{q} \circ \pi_{p(i)} \circ \gamma_{f}=\lambda_{q} \circ \Gamma_{f} \circ \pi_{p(i)}$.

Because $p$ is a permutation, this means that $\Gamma_{f} \circ \lambda_{q}=\lambda_{q} \circ \Gamma_{f}$ on every $\operatorname{im} \pi_{i}$.

Remark 10.9. To explain when the homomorphism $\sigma_{j} \mapsto \widetilde{\sigma}_{j}$ is faithful, we can make the following definition: we say that $1 \leq i \leq N$ is a slave for the network $\Sigma$ if there are no $1 \leq j \leq n$ and $1 \leq k \leq N$ so that $\sigma_{j}(k)=i$. Thus, a slave is a cell that does not act as input for any other cell, not even for itself. The point of this definition is the following:

Proposition 10.10. If $\Sigma$ has no slaves, then $\sigma_{j} \mapsto \widetilde{\sigma}_{j}$ is a faithful homomorphism.

Proof. The relation $\widetilde{\sigma}_{j_{1}}=\widetilde{\sigma}_{j_{2}}$ means that $\sigma_{j_{1}} \circ \sigma_{k}=\sigma_{j_{2}} \circ \sigma_{k}$ for all $k$. This implies in particular that $\sigma_{j_{1}}=\sigma_{j_{2}}$ on $\operatorname{im} \sigma_{k}$ for all $k$. But if $\Sigma$ is free of slaves, then $\bigcup_{j=1}^{n} \operatorname{im} \sigma_{j}=\{1, \ldots, N\}$. Hence, $\sigma_{j_{1}}=\sigma_{j_{2}}$.

If a network has slaves, then we can reduce it until no slaves remain. This works as follows: first of all, we remove any slave from the network. Because slaves do not affect the dynamics of other cells, this can be done without any effect on the network dynamics. Removing slaves may create new slaves: these are the cells that acted as inputs only for the original slaves. These new slaves can also be removed, etc., until a network free of slaves remains.

The remaining network may not be defined unambiguously, because some of the maps in $\Sigma$ may coincide after the removal of the slaves. This happens when distinct maps in $\Sigma$ differ only at slaves. Such maps can be identified though, while $f$ must be redefined. In this way, we produce an unambiguous network that is free of slaves. For such a network $\gamma_{f}$, the corresponding $\Gamma_{f}$ is a true fundamental network.

\section{SOME EXAMPLES AND THEIR NORMAL FORMS}

In this section we illustrate the methods and results of this paper by computing the normal forms of two coupled cell networks. Keeping things simple, we restrict our attention to synchrony breaking steady state bifurcations in one parameter families of networks with one-dimensional cells. 
11.1. A skew product network. In the first example, we consider the homogeneous skew product differential equations

$$
\begin{aligned}
& \dot{x}_{1}=f\left(x_{1}, x_{1} ; \lambda\right), \\
& \dot{x}_{2}=f\left(x_{2}, x_{1} ; \lambda\right) .
\end{aligned}
$$

Here $x_{1}, x_{2} \in \mathbb{R}$ and $f: \mathbb{R}^{2} \times \mathbb{R} \rightarrow \mathbb{R}$. As usual, we will denote the right hand side of (11.26) by $\gamma_{f}\left(x_{1}, x_{2} ; \lambda\right)$, and we will henceforth assume that

$$
\gamma_{f}(0,0 ; 0)=0 \text { and } D_{x} \gamma_{f}(0,0 ; 0) \text { is not invertible. }
$$

This means that at the parameter value $\lambda=0$, the origin $\left(x_{1}, x_{2}\right)=(0,0)$ is a fully synchronous equilibrium point of (11.26) that undergoes a steady state bifurcation. We wish to study the generic nature of this bifurcation. So let us write

$$
f_{0,0}\left(X_{1}, X_{2}\right)=D_{X} f(0,0 ; 0)\left(X_{1}, X_{2}\right)=a_{1} X_{1}+a_{2} X_{2} \text { with } a_{1}, a_{2} \in \mathbb{R} .
$$

With this notation, we have that

$$
\text { mat } D_{x} \gamma_{f}(0,0 ; 0)=\left(\begin{array}{cc}
a_{1}+a_{2} & 0 \\
a_{2} & a_{1}
\end{array}\right) .
$$

We remark that this linearization matrix is semisimple and moreover that a steady state bifurcation occurs when one of its eigenvalues $a_{1}+a_{2}$ or $a_{1}$ vanishes.

The obvious but important remark is now that equations (11.26) define a semigroup coupled cell network. The corresponding semigroup consists of $\sigma_{1}$ and $\sigma_{2}$, where

$$
\sigma_{1}(1)=1, \sigma_{1}(2)=2 \text { and } \sigma_{2}(1)=1, \sigma_{2}(2)=1 .
$$

We depicted this network in Figure 6 .

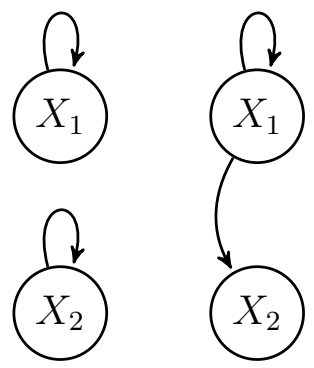

Figure 6. A homogeneous skew product network.

The composition table of $\left\{\sigma_{1}, \sigma_{2}\right\}$ reads

\begin{tabular}{c|cc}
$\circ$ & $\sigma_{1}$ & $\sigma_{2}$ \\
\hline$\sigma_{1}$ & $\sigma_{1}$ & $\sigma_{2}$ \\
$\sigma_{2}$ & $\sigma_{2}$ & $\sigma_{2}$
\end{tabular}.

From this table, we can read that

$$
A_{\sigma_{1}}\left(X_{1}, X_{2}\right)=\left(X_{1}, X_{2}\right), A_{\sigma_{2}}\left(X_{1}, X_{2}\right)=\left(X_{2}, X_{2}\right),
$$

and hence that the symbolic Lie bracket of this network is given by

$$
\begin{aligned}
{[f, g]_{\Sigma}\left(X_{1}, X_{2}\right) } & =D_{1} f\left(X_{1}, X_{2}\right) g\left(X_{1}, X_{2}\right)+D_{2} f\left(X_{1}, X_{2}\right) g\left(X_{2}, X_{2}\right) \\
& -D_{1} g\left(X_{1}, X_{2}\right) f\left(X_{1}, X_{2}\right)-D_{2} g\left(X_{1}, X_{2}\right) f\left(X_{2}, X_{2}\right) .
\end{aligned}
$$


Since $D_{x} \gamma_{f}(0,0 ; 0)$ is semisimple, so is $\operatorname{ad}_{f_{0,0}}^{\Sigma}: P^{k, l} \rightarrow P^{k, l}$ for every $k \geq-1$ and $l \geq 0$. Its kernel determines the normal form of $f$. It only requires a little computation to check that $\operatorname{ad}_{f_{0,0}}^{\Sigma}:\left\{\begin{array}{lll}\left(X_{1}-X_{2}\right)^{\alpha} X_{2}^{\beta} & \mapsto\left[(1-\alpha) a_{1}-\beta\left(a_{1}+a_{2}\right)\right]\left(X_{1}-X_{2}\right)^{\alpha} X_{2}^{\beta} \\ X_{2}^{\beta} & \mapsto(1-\beta)\left(a_{1}+a_{2}\right) X_{2}^{\beta} & \text { for } \alpha \geq 1 \text { and } \beta \geq 0,\end{array}\right.$

This formula nicely confirms that $\operatorname{ad}_{f_{0,0}}^{\Sigma}$ is semisimple. We now consider the two different codimension one cases:

1. When $a_{1}+a_{2}=0$ but $a_{1} \neq 0$, then the kernel of $D_{x} \gamma_{f}(0,0 ; 0)$ is tangent to the synchrony space $\left\{x_{1}=x_{2}\right\}$. In this case, the kernel of $\operatorname{ad}_{f_{0,0}}^{\Sigma}$ is spanned by elements of the form $\left(X_{1}-X_{2}\right) X_{2}^{\beta}$ and $X_{2}^{\beta}$, where $\beta \geq 0$. Thus, the general normal form of $f$ is

$$
\bar{f}\left(X_{1}, X_{2} ; \lambda\right)=\left(X_{1}-X_{2}\right) F\left(X_{2} ; \lambda\right)+G\left(X_{2} ; \lambda\right),
$$

with $F\left(X_{2} ; \lambda\right)=A(\lambda)+\mathcal{O}\left(X_{2}\right), G\left(X_{2} ; \lambda\right)=B(\lambda)+C(\lambda) X_{2}+D(\lambda) X_{2}^{2}+$ $\mathcal{O}\left(X_{2}^{3}\right)$ and $A(0)=a_{1}, B(0)=C(0)=0$. The normal form equations of motion become

$$
\begin{aligned}
& \dot{x}_{1}=G\left(x_{1} ; \lambda\right), \\
& \dot{x}_{2}=G\left(x_{1} ; \lambda\right)+\left(x_{2}-x_{1}\right) F\left(x_{1} ; \lambda\right) .
\end{aligned}
$$

This implies first of all that the stationary points of the normal form satisfy $x_{1}=x_{2}$ and secondly that $x_{1}$ solves the equation $G\left(x_{1} ; \lambda\right)=$ $B(\lambda)+C(\lambda) x_{1}+D(\lambda) x_{1}^{2}+\mathcal{O}\left(x_{1}^{3}\right)=0$. Under the generic conditions that $B^{\prime}(0), D(0) \neq 0$, we thus find the saddle node branches

$$
x_{1}=x_{2}= \pm \sqrt{\left(-B^{\prime}(0) / D(0)\right) \lambda}+\mathcal{O}(\lambda)
$$

of synchronous steady states. A straightforward stability analysis reveals that one of these branches consists of equilibria that are linearly stable in the direction of the synchrony space, while the other branch consists of unstable points. We remark that the saddle node bifurcation is also generic in codimension one in the context of vector fields without any special structure.

2. When $a_{1}+a_{2} \neq 0$ and $a_{1}=0$, then the kernel of $\operatorname{ad}_{f_{0,0}}^{\Sigma}$ is spanned by elements of the form $\left(X_{1}-X_{2}\right)^{\alpha}$, where $\alpha \geq 1$, and the element $X_{2}$. Hence the general normal form of $f$ is given by

$$
\bar{f}\left(X_{1}, X_{2} ; \lambda\right)=\left(X_{1}-X_{2}\right) F\left(X_{1}-X_{2} ; \lambda\right)+A(\lambda) X_{2},
$$

with $F\left(X_{1}-X_{2} ; \lambda\right)=B(\lambda)+C(\lambda)\left(X_{1}-X_{2}\right)+\mathcal{O}\left(X_{1}-X_{2}\right)^{2}$ and $A(0)=$ $a_{2}, B(0)=0$. The normal form differential equations are

$$
\begin{aligned}
& \dot{x}_{1}=A(\lambda) x_{1}, \\
& \dot{x}_{2}=A(\lambda) x_{1}+\left(x_{2}-x_{1}\right) F\left(x_{2}-x_{1} ; \lambda\right) .
\end{aligned}
$$

This implies that the stationary points of the normal form satisfy $x_{1}=0$, while either $x_{2}=0$ or $x_{2}$ solves the equation $F\left(x_{2} ; \lambda\right)=B(\lambda)+C(\lambda) x_{2}+$ $\mathcal{O}\left(x_{2}^{2}\right)=0$. Under the generic conditions that $B^{\prime}(0), C(0) \neq 0$, we thus find the two steady state branches

$$
x_{1}=x_{2}=0 \text { and } x_{1}=0, x_{2}=\left(-B^{\prime}(0) / C(0)\right) \lambda+\mathcal{O}\left(\lambda^{2}\right) .
$$


These branches exchange stability when they cross. This means that the normal form displays a synchrony breaking transcritical bifurcation. Such a bifurcation is not generic in codimension one in the context of vector fields without any special structure, and is hence forced by the network structure. More precisely, it follows from the presence of the invariant synchrony space.

11.2. A nilpotent feed-forward network. Next, we consider differential equations with the network structure defined in Example 2.4.

$$
\begin{aligned}
& \dot{x}_{1}=f\left(x_{1}, x_{1}, x_{1} ; \lambda\right), \\
& \dot{x}_{2}=f\left(x_{2}, x_{1}, x_{1} ; \lambda\right), \\
& \dot{x}_{3}=f\left(x_{3}, x_{2}, x_{1} ; \lambda\right) .
\end{aligned}
$$

Here $x_{1}, x_{2}, x_{3} \in \mathbb{R}$ and $f: \mathbb{R}^{3} \times \mathbb{R} \rightarrow \mathbb{R}$. Again, let us write

$$
\begin{aligned}
f_{0,0}\left(X_{1}, X_{2}, X_{3}\right) & =D_{X} f(0,0,0 ; 0)\left(X_{1}, X_{2}, X_{3}\right) \\
& =a_{1} X_{1}+a_{2} X_{2}+a_{3} X_{3} \text { for } a_{1}, a_{2}, a_{3} \in \mathbb{R} .
\end{aligned}
$$

Then it holds that

$$
\operatorname{mat} D_{x} \gamma_{f}(0,0,0 ; 0)=\left(\begin{array}{rrr}
a_{1}+a_{2}+a_{3} & 0 & 0 \\
a_{2}+a_{3} & a_{1} & 0 \\
a_{3} & a_{2} & a_{1}
\end{array}\right) .
$$

This shows that a steady state bifurcation takes place when either $a_{1}+a_{2}+a_{3}=0$ or $a_{1}=0$. Moreover, the linearization matrix is not semisimple. In fact, its SNdecomposition reads

$$
\left(\begin{array}{rrr}
a_{1}+a_{2}+a_{3} & 0 & 0 \\
a_{2}+a_{3} & a_{1} & 0 \\
a_{3} & a_{2} & a_{1}
\end{array}\right)=\left(\begin{array}{rrr}
a_{1}+a_{2}+a_{3} & 0 & 0 \\
a_{2}+a_{3} & a_{1} & 0 \\
a_{2}+a_{3} & 0 & a_{1}
\end{array}\right)+\left(\begin{array}{rrr}
0 & 0 & 0 \\
0 & 0 & 0 \\
-a_{2} & a_{2} & 0
\end{array}\right) .
$$

As a consequence, we should accordingly decompose $f_{0,0}$ as

$$
f_{0,0}=f_{0,0}^{S}+f_{0,0}^{N},
$$

where

$$
f_{0,0}^{S}\left(X_{1}, X_{2}, X_{3}\right)=a_{1} X_{1}+\left(a_{2}+a_{3}\right) X_{3}, f_{0,0}^{N}\left(X_{1}, X_{2}, X_{3}\right)=a_{2}\left(X_{2}-X_{3}\right) .
$$

Recalling that for this network the expression for the symbolic bracket is given in Example 5.3, it again requires a little computation to find that

$$
\operatorname{ad}_{f_{0,0}^{S}}:\left\{\begin{array}{llr}
X_{3}^{\gamma} & \mapsto(1-\gamma)\left(a_{1}+a_{2}+a_{3}\right) X_{3}^{\gamma} & \text { for } \gamma \geq 0, \\
\left(X_{1}-X_{3}\right)^{\alpha}\left(X_{2}-X_{3}\right)^{\beta} X_{3}^{\gamma} & \mapsto & \\
{\left[(1-\alpha-\beta) a_{1}-\gamma\left(a_{1}+a_{2}+a_{3}\right)\right]\left(X_{1}-X_{3}\right)^{\alpha}\left(X_{2}-X_{3}\right)^{\beta} X_{3}^{\gamma}} & \text { for } \alpha+\beta \geq 1, \\
& \gamma \geq 0 .
\end{array}\right.
$$

and similarly that

$$
\operatorname{ad}_{f_{0,0}^{N}}:\left\{\begin{array}{lll}
\left(X_{1}-X_{3}\right)^{\alpha}\left(X_{2}-X_{3}\right)^{\beta} X_{3}^{\gamma} & \mapsto & \\
-\alpha a_{2}\left(X_{1}-X_{3}\right)^{\alpha-1}\left(X_{2}-X_{3}\right)^{\beta+1} X_{3}^{\gamma} & \text { for } \alpha, \beta \geq 1, \gamma \geq 0, \\
\left(X_{1}-X_{3}\right)^{\alpha} X_{3}^{\gamma} & \mapsto & \\
a_{2}\left(X_{2}-X_{3}\right)^{\alpha} X_{3}^{\gamma}-\alpha a_{2}\left(X_{1}-X_{3}\right)^{\alpha-1}\left(X_{2}-X_{3}\right) X_{3}^{\gamma} & \text { for } \alpha \geq 1, \gamma \geq 0, \\
\left(X_{2}-X_{3}\right)^{\beta} X_{3}^{\gamma} & \mapsto 0 & \text { for } \beta, \gamma \geq 0
\end{array}\right.
$$


Once more, we now consider the two codimension one cases:

1. If $a_{1}+a_{2}+a_{3}=0$ and $a_{1} \neq 0$, then the kernel of $\operatorname{ad}_{f_{0,0}}^{\Sigma}$ is spanned by terms

$$
\left(X_{1}-X_{3}\right) X_{3}^{\gamma},\left(X_{2}-X_{3}\right) X_{3}^{\gamma} \text { and } X_{3}^{\gamma} \text { with } \gamma \geq 0 \text {. }
$$

One checks that $\operatorname{ad}_{f_{0,0}^{N}}$ vanishes on this kernel, so the general normal form of $f$ is

$\bar{f}\left(X_{1}, X_{2}, X_{3} ; \lambda\right)=\left(X_{1}-X_{3}\right) F\left(X_{3} ; \lambda\right)+\left(X_{2}-X_{3}\right) G\left(X_{3} ; \lambda\right)+H\left(X_{3} ; \lambda\right)$,

where $F\left(X_{3} ; \lambda\right)=A(\lambda)+\mathcal{O}\left(X_{3}\right), G\left(X_{3} ; \lambda\right)=B(\lambda)+\mathcal{O}\left(X_{3}\right), H\left(X_{3} ; \lambda\right)=$ $C(\lambda)+D(\lambda) X_{3}+E(\lambda) X_{3}^{2}+\mathcal{O}\left(X_{3}^{3}\right)$ and $A(0)=a_{1}, B(0)=a_{2}, C(0)=$ $D(0)=0$. The normal form equations of motion are

$$
\begin{aligned}
& \dot{x}_{1}=H\left(x_{1} ; \lambda\right), \\
& \dot{x}_{2}=\left(x_{2}-x_{1}\right) F\left(x_{1} ; \lambda\right)+H\left(x_{1} ; \lambda\right), \\
& \dot{x}_{3}=\left(x_{3}-x_{1}\right) F\left(x_{1} ; \lambda\right)+\left(x_{2}-x_{1}\right) G\left(x_{1} ; \lambda\right)+H\left(x_{1} ; \lambda\right) .
\end{aligned}
$$

It follows that the steady states of the normal form satisfy $x_{1}=x_{2}=x_{3}$, where $x_{1}$ satisfies $H\left(x_{1} ; \lambda\right)=C(\lambda)+D(\lambda) x_{1}+E(\lambda) x_{1}^{2}+\mathcal{O}\left(x_{1}^{3}\right)=0$. Under the generic conditions that $C^{\prime}(0), E(0) \neq 0$, this yields the fully synchronous saddle node branches

$$
x_{1}=x_{2}=x_{3}= \pm \sqrt{-\left(C^{\prime}(0) / E(0)\right) \lambda}+\mathcal{O}(\lambda) .
$$

Again, one of these branches is stable and the other one is unstable in the direction of the maximal synchrony space.

2. When $a_{1}=0, a_{2} \neq 0$ and $a_{1}+a_{2}+a_{3} \neq 0$, then $\operatorname{ker}_{f_{0,0}^{S}}^{\Sigma}$ is spanned by the elements

$$
X_{3} \text { and }\left(X_{1}-X_{3}\right)^{\alpha}\left(X_{2}-X_{3}\right)^{\beta} \text { with } \alpha+\beta \geq 1 .
$$

This time the action of $\operatorname{ad}_{f_{0,0}^{N}}^{\Sigma}$ on $\operatorname{ker}_{\operatorname{ad}_{0_{0,0}^{S}}}^{\Sigma}$ is nontrivial. The only terms in the kernel that are not in im $\operatorname{ad}_{f_{0,0}^{N}}$ are actually those of the form

$$
\left(X_{1}-X_{3}\right)^{\alpha}, X_{2}-X_{3} \text { and } X_{3} \text {, with } \alpha \geq 1 \text {. }
$$

This means that the general normal form of $f$ is

$$
\begin{aligned}
& \bar{f}\left(X_{1}, X_{2}, X_{3} ; \lambda\right)=\left(X_{1}-X_{3}\right) F\left(X_{1}-X_{3}\right)+A(\lambda)\left(X_{2}-X_{3}\right)+B(\lambda) X_{3}, \\
& \text { where } F\left(X_{1}-X_{3}\right)=C(\lambda)+D(\lambda)\left(X_{1}-X_{3}\right)+\mathcal{O}\left(X_{1}-X_{3}\right)^{2} \text { and } A(0)= \\
& a_{2}, B(0)=a_{1}+a_{2}+a_{3}, C(0)=0 . \text { This gives the equations of motion } \\
& \quad \dot{x}_{1}=B(\lambda) x_{1}, \\
& \dot{x}_{2}=B(\lambda) x_{1}+\left(x_{2}-x_{1}\right) F\left(x_{2}-x_{1} ; \lambda\right), \\
& \dot{x}_{3}=B(\lambda) x_{1}+A(\lambda)\left(x_{2}-x_{1}\right)+\left(x_{3}-x_{1}\right) F\left(x_{3}-x_{1} ; \lambda\right) .
\end{aligned}
$$

Under the generic assumption that $C^{\prime}(0), D(0) \neq 0$, we now find three branches of steady states:

$$
\begin{aligned}
& x_{1}=x_{2}=x_{3}=0, \\
& x_{1}=x_{2}=0, x_{3}=-\left(C^{\prime}(0) / D(0)\right) \lambda+\mathcal{O}\left(\lambda^{2}\right), \\
& x_{1}=0, x_{2}=-\left(C^{\prime}(0) / D(0)\right) \lambda+\mathcal{O}\left(\lambda^{2}\right), x_{3}= \pm \sqrt{\left(a_{2} C^{\prime}(0) / D(0)^{2}\right) \lambda}+\mathcal{O}(\lambda) .
\end{aligned}
$$


This means that our normal form equations undergo a very particular synchrony breaking steady state bifurcation that comprises a fully synchronous trivial branch, a partially synchronous transcritical branch and fully nonsynchronous saddle node branches. The solutions on these branches exchange stability in a specific way, as for example depicted in Figure 7

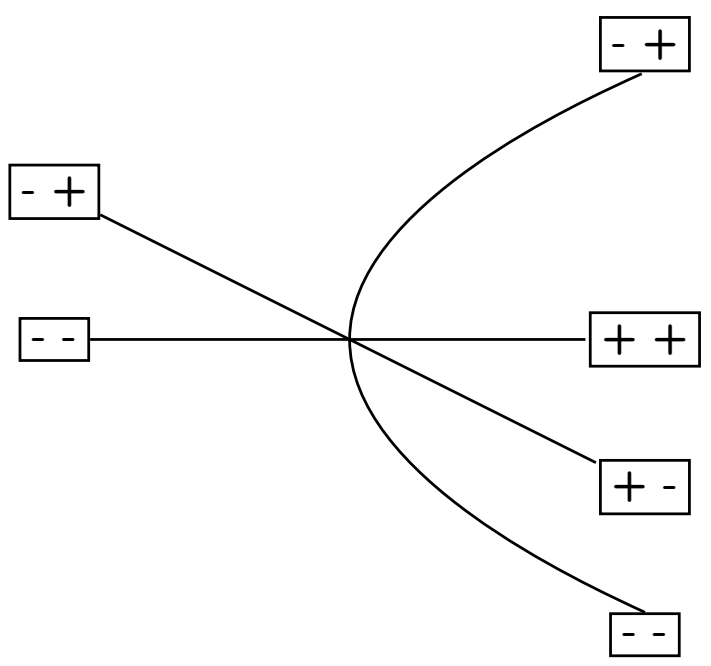

FiguRE 7. Bifurcation diagram of a codimension one steady state bifurcation in the normal form of a three cell feedforward network. Pluses and minuses refer to positive and negative eigenvalues in the eigendirections other than the maximal synchrony space. This figure depicts the solutions of formula (11.30) in the case $a_{2}, C^{\prime}(0), D(0)>0$.

\section{Colored COUPLED CELl Networks}

In this final section, we describe how our results on homogeneous coupled cell networks generalize to certain nonhomogeneous coupled cell networks. So let us imagine a coupled cell network with cells of different types. We will refer to the different types of cells as colors.

More precisely, let us assume that there are $1 \leq C<\infty$ colors and that for every color $1 \leq c \leq C$ there are precisely $N_{c}$ cells of color $c$. We label the cells of color $c$ by $1 \leq i \leq N_{c}$ and assume that the state of the $i$-th cell of color $c$ is described by $x_{i}^{(c)} \in V_{c}$, where $V_{c}$ is a linear space that depends on $c$.

We furthermore assume that the discrete- or continuous-time evolution of $x_{i}^{(c)}$ is determined by precisely $n_{(1, c)}$ cells of color 1 , by $n_{(2, c)}$ cells of color 2 , etc. This assumption is made precise in Definition 12.1 below that, although lengthy, is a straightforward generalization of Definition 2.1.

Definition 12.1. For every $1 \leq c, d \leq C$ and every $1 \leq j \leq n_{(d, c)}$, assume there is a map

$$
\sigma_{j}^{(d, c)}:\left\{1, \ldots, N_{c}\right\} \rightarrow\left\{1, \ldots, N_{d}\right\}
$$


We denote the collection of these maps by

$$
\Sigma:=\left\{\sigma_{1}^{(1,1)}, \ldots, \sigma_{n_{(1,1)}^{(1,1)}} ; \ldots ; \sigma_{1}^{(C, C)}, \ldots, \sigma_{n_{(C, C)}^{(C, C)}}^{(C,}\right\} .
$$

Next, we define for all $1 \leq c \leq C$ and $1 \leq i \leq N_{c}$ the maps

$$
\begin{gathered}
\pi_{i}^{(c)}: V_{1}^{N_{1}} \times \ldots \times V_{C}^{N_{C}} \rightarrow V_{1}^{n_{1, c}} \times \ldots \times V_{C}^{n_{C, c}} \text { by } \\
\pi_{i}^{(c)}\left(x^{(1)} ; \ldots ; x^{(C)}\right):=\left(x_{\sigma_{1}^{(1, c)}(i)}^{(1)}, \ldots, x_{\sigma_{n_{(1, c)}^{(1, c)}}^{(1)}(i)} ; \ldots ; x_{\sigma_{1}^{(C, c)}(i)}^{(C)}, \ldots, x_{\sigma_{n(C, c)}^{(C)}(i)}^{(C)}\right) .
\end{gathered}
$$

Now assume that $f=\left(f^{(1)}, \ldots, f^{(C)}\right)$ is a collection of functions with

$$
f^{(c)}: V_{1}^{n_{(1, c)}} \times \ldots \times V_{C}^{n_{(C, c)}} \rightarrow V_{c} .
$$

Then we define $\gamma_{f}: V_{1}^{N_{1}} \times \ldots \times V_{C}^{N_{C}} \rightarrow V_{1}^{N_{1}} \times \ldots \times V_{C}^{N_{C}}$ by

$$
\left(\gamma_{f}\right)_{i}^{(c)}:=f^{(c)} \circ \pi_{i}^{(c)} \text { for all } 1 \leq c \leq C \text { and } 1 \leq i \leq N_{c} .
$$

We say that $\gamma_{f}$ is a colored coupled cell network map/vector field subject to $\Sigma$.

It is important to note that only the compositions

$$
\sigma_{j_{1}}^{e, d} \circ \sigma_{j_{2}}^{d, c}:\left\{1, \ldots, N_{c}\right\} \rightarrow\left\{1, \ldots, N_{e}\right\}
$$

are sensibly defined. This inspires the following definition:

Definition 12.2. We say that $\Sigma$ is a semigroupoid if for every $1 \leq c, d, e \leq C$ and every $1 \leq j_{1} \leq n_{d, c}$ and $1 \leq j_{2} \leq n_{e, d}$ there is precisely one $1 \leq j_{3} \leq n_{e, c}$ such that

$$
\sigma_{j_{1}}^{(e, d)} \circ \sigma_{j_{2}}^{(d, c)}=\sigma_{j_{3}}^{(e, c)} \text {. }
$$

When a collection $\Sigma$ as in Definition 12.1 is not a semigroupoid, then it generates one: the smallest semigroupoid $\Sigma^{\prime}$ containing $\Sigma$.

Example 12.3. The completely general $C$-dimensional differential equation

$$
\dot{x}^{(c)}=f^{(c)}\left(x^{(1)} ; \ldots ; x^{(C)}\right) \text { for } 1 \leq c \leq C \text { and } x^{(c)} \in V_{c}
$$

is an example of a colored coupled cell network with $C$ colors and one cell of each color. The elements of $\Sigma=\left\{\sigma_{1}^{(1,1)} ; \ldots ; \sigma_{C}^{(C, C)}\right\}$ are all defined by $\sigma_{j}^{(d, c)}(1)=1$. They obviously form a semigroupoid.

Example 12.4. The general 2-dimensional skew product differential equation

$$
\begin{aligned}
& \dot{x}^{(1)}=f^{(1)}\left(x^{(1)}\right), \\
& \dot{x}^{(2)}=f^{(2)}\left(x^{(1)} ; x^{(2)}\right)
\end{aligned}
$$

with $x^{(1)} \in V_{1}$ and $x^{(2)} \in V_{2}$ is an example of a colored coupled cell network with two colors and one cell of each color. The elements of $\Sigma=\left\{\sigma_{1}^{(1,1)} ; \sigma_{1}^{(1,2)}, \sigma_{1}^{(2,2)}\right\}$ are all defined by $\sigma_{1}^{(d, c)}(1)=1$ and thus form a semigroupoid. See Figure 8 .

Example 12.5. The 3-dimensional differential equation

$$
\begin{aligned}
& \dot{x}_{1}^{(1)}=f^{(1)}\left(x_{2}^{(1)} ; x_{1}^{(2)}\right), \\
& \dot{x}_{2}^{(1)}=f^{(1)}\left(x_{2}^{(1)} ; x_{1}^{(2)}\right), \\
& \dot{x}_{1}^{(2)}=f^{(2)}\left(x_{2}^{(1)}\right)
\end{aligned}
$$



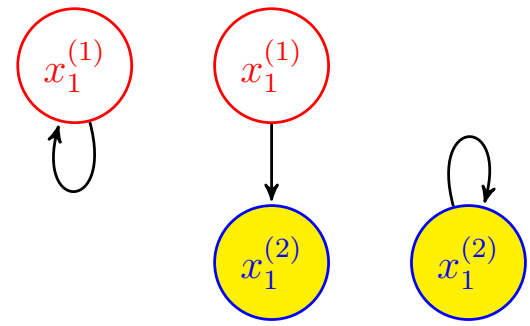

FiguRE 8. A colored skew product network.

with $x_{1}^{(1)}, x_{2}^{(1)} \in V_{1}$ and $x_{1}^{(2)} \in V_{2}$ is an example of a colored coupled cell network with two colors: two cells of color 1 and one cell of color 2 . Here, $\Sigma=$ $\left\{\sigma_{1}^{(1,1)}, \sigma_{1}^{(1,2)} ; \sigma_{1}^{(2,1)}\right\}$, where these maps are defined by

$$
\sigma_{1}^{(1,1)}(1)=2, \sigma_{1}^{(1,1)}(2)=2 ; \sigma_{1}^{(1,2)}(1)=2 ; \sigma_{1}^{(2,1)}(1)=1, \sigma_{1}^{(2,1)}(2)=1 .
$$

Again, one quickly checks that $\Sigma$ is a semigroupoid. See Figure 9 .
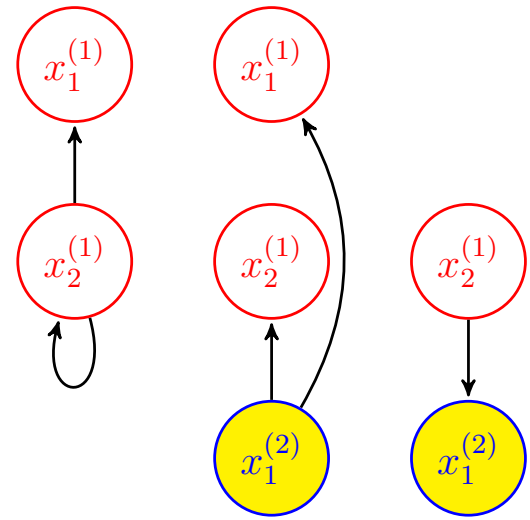

FiguRE 9. An example of a colored network with three cells of two colors.

Under the condition that $\Sigma$ is a semigroupoid, all results of this paper on Lie algebras and normal forms can be generalized to colored coupled cell networks. As an illustration, we state a few facts here without proof.

Theorem 12.6. If $\Sigma$ is a semigroupoid, then for each $\sigma_{j}^{(d, c)} \in \Sigma$ there is a unique linear map

$$
A_{\sigma_{j}^{(d, c)}}: V_{1}^{n_{(1, c)}} \times \ldots \times V_{C}^{n_{(C, c)}} \rightarrow V_{1}^{n_{(1, d)}} \times \ldots \times V_{C}^{n_{(C, d)}}
$$

such that for all $1 \leq i \leq N_{c}$ it holds that

$$
A_{\sigma_{j}^{(d, c)}} \circ \pi_{i}^{(c)}=\pi_{\sigma_{j}^{(d, c)}(i)}^{(d)} .
$$

These maps satisfy the relations $A_{\sigma_{j_{1}}^{(e, d)}} \circ A_{\sigma_{j_{2}}^{(d, c)}}=A_{\sigma_{j_{1}}^{(e, d)} \circ \sigma_{j_{2}}^{(d, c)}}$ and thus form $a$ representation of the semigroupoid $\Sigma$. 
Theorem 12.7. If $\Sigma$ is a semigroupoid, then

$$
\gamma_{f} \circ \gamma_{g}=\gamma_{f \circ \Sigma} g
$$

in which $\left(f \circ \circ_{\Sigma} g\right)^{(c)}$ is equal to

$$
\begin{aligned}
f^{(c)} \circ\left(g^{(1)} \circ A_{\sigma_{1}^{(1, c)}} \times \ldots \times g^{(1)} \circ A_{\sigma_{n(1, c)}^{(1, c)}}\right. & \times \ldots \times g^{(C)} \\
& \left.\circ A_{\sigma_{1}^{(C, c)}} \times \ldots \times g^{(C)} \circ A_{\sigma_{n(C, c)}^{(C, c)}}\right) .
\end{aligned}
$$

Theorem 12.8. If $\Sigma$ is a semigroupoid, then

$$
\left[\gamma_{f}, \gamma_{g}\right]=\gamma_{[f, g]_{\Sigma}}
$$

in which $[f, g]_{\Sigma}^{(c)}$ equals

$$
\sum_{d} \sum_{j}\left(D_{X_{j}^{(d)}} f^{(c)} \cdot\left(g^{(d)} \circ A_{\sigma_{j}^{(d, c)}}\right)-D_{X_{j}^{(d)}} g^{(c)} \cdot\left(f^{(d)} \circ A_{\sigma_{j}^{(d, c)}}\right)\right) .
$$

In turn, Theorem 12.7 can be used to prove normal form theorems for colored coupled cell networks. That is, the theorems of Sections 6 and 9 remain true with the word "semigroup" replaced by "semigroupoid".

We conclude with two results that say that the network symmetries and the robust synchrony spaces of a network remain unchanged by the semigroupoid extension.

Lemma 12.9. Let $\Sigma$ be as in Definition 12.1, not necessarily forming a semigroupoid, and let $p$ be a permutation of the cells so that the restriction $p:\left\{1, \ldots, N_{c}\right\}$ $\rightarrow\left\{1, \ldots, N_{c}\right\}$ preserves the cells of each color. We say that $p$ is a network symmetry if

$$
p \circ \sigma_{j}^{(d, c)}=\sigma_{j}^{(d, c)} \circ p \text { for all } 1 \leq c, d \leq C \text { and all } 1 \leq j \leq n_{(d, c)} .
$$

This means that $\lambda_{p}$ sends orbits of $\gamma_{f}$ to orbits of $\gamma_{f}$.

Then the collection of network symmetries of $\Sigma$ is the same as the collection of network symmetries of the semigroupoid $\Sigma^{\prime}$ generated by $\Sigma$.

Lemma 12.10. Let $\Sigma$ be as in Definition [12.1, not necessarily forming a semigroupoid, and let $P=\left\{P^{(1)}, \ldots, P^{(C)}\right\}$ be a collection of partitions; i.e. for all $1 \leq c \leq C$, we have that $P^{(c)}=\left\{P_{1}^{(c)}, \ldots, P_{r_{c}}^{(c)}\right\}$ is a partition of $\left\{1, \ldots, N_{c}\right\}$. Then the following are equivalent:

i) The collection of partitions is balanced; i.e. for all $1 \leq c, d \leq C$, all $1 \leq j \leq$ $n_{(d, c)}$ and all $1 \leq k_{1} \leq r_{c}$ there exists a $1 \leq k_{2} \leq r_{d}$ so that $\sigma_{j}^{(d, c)}\left(P_{k_{1}}^{(c)}\right) \subset$ $P_{k_{2}}^{(d)}$.

ii) The subspace

$$
\begin{aligned}
\operatorname{Syn}_{P}:=\left\{x \in V^{N_{1}} \times \ldots \times V^{N_{C}} \mid x_{i_{1}}^{(c)}=x_{i_{2}}^{(c)} \text { when } i_{1} \text { and } i_{2}\right. \\
\text { are in the same element of } \left.P^{(c)}\right\}
\end{aligned}
$$

is a robust synchrony space for the networks subject to $\Sigma$.

The collection of robust synchrony spaces of $\Sigma$ is the same as the collection of robust synchrony spaces of the semigroupoid $\Sigma^{\prime}$ generated by $\Sigma$. 


\section{REFERENCES}

[1] Manuela A. D. Aguiar, Ana Paula S. Dias, Martin Golubitsky, and Maria da Conceição A. Leite, Bifurcations from regular quotient networks: a first insight, Phys. D 238 (2009), no. 2, 137-155, DOI 10.1016/j.physd.2008.10.006. MR2516333 (2011m:37079)

[2] Fernando Antoneli, Ana Paula Dias, Martin Golubitsky, and Yunjiao Wang, Patterns of synchrony in lattice dynamical systems, Nonlinearity 18 (2005), no. 5, 2193-2209, DOI 10.1088/0951-7715/18/5/016. MR2164738(2006k:37223)

[3] Fernando Antoneli, Ana Paula S. Dias, Martin Golubitsky, and Yunjiao Wang, Synchrony in lattice differential equations, Some topics in industrial and applied mathematics, Ser. Contemp. Appl. Math. CAM, vol. 8, Higher Ed. Press, Beijing, 2007, pp. 43-56, DOI 10.1142/9789812709356_0003. MR2381908 (2009e:37076)

[4] Fernando Antoneli, Ana Paula S. Dias, and Rui C. Paiva, Hopf bifurcation in coupled cell networks with interior symmetries, SIAM J. Appl. Dyn. Syst. 7 (2008), no. 1, 220-248, DOI 10.1137/070684604. MR2399564 (2009h:37110)

[5] Fernando Antoneli and Ian Stewart, Symmetry and synchrony in coupled cell networks. I. Fixed-point spaces, Internat. J. Bifur. Chaos Appl. Sci. Engrg. 16 (2006), no. 3, 559-577, DOI 10.1142/S0218127406015167. MR2228833 (2008k:37059)

[6] Fernando Antoneli and Ian Stewart, Symmetry and synchrony in coupled cell networks. II. Group networks, Internat. J. Bifur. Chaos Appl. Sci. Engrg. 17 (2007), no. 3, 935-951, DOI 10.1142/S0218127407017641. MR2324988(2008k:37060)

[7] Richard Cushman and Jan A. Sanders, Splitting algorithm for nilpotent normal forms, Dynam. Stability Systems 2 (1987), no. 3-4, 235-246. MR954291 (89i:58032)

[8] Ana Paula S. Dias and Jeroen S. W. Lamb, Local bifurcation in symmetric coupled cell networks: linear theory, Phys. D 223 (2006), no. 1, 93-108, DOI 10.1016/j.physd.2006.08.014. MR2304828(2008d:37089)

[9] Ana Paula S. Dias and Rui C. Paiva, Hopf bifurcation in coupled cell networks with abelian symmetry, Bol. Soc. Port. Mat. Special Issue (2010), 110-115. MR2839753 (2012g:37097)

[10] Benoit Dionne, Martin Golubitsky, and Ian Stewart, Coupled cells with internal symmetry. I. Wreath products, Nonlinearity 9 (1996), no. 2, 559-574, DOI 10.1088/0951-7715/9/2/016. MR $1384492(97 \mathrm{j}: 58110)$

[11] Benoit Dionne, Martin Golubitsky, and Ian Stewart, Coupled cells with internal symmetry. II. Direct products, Nonlinearity 9 (1996), no. 2, 575-599, DOI 10.1088/0951-7715/9/2/017. MR $1384493(97 \mathrm{j}: 58111)$

[12] J. J. Duistermaat, Bifurcation of periodic solutions near equilibrium points of Hamiltonian systems, Bifurcation theory and applications (Montecatini, 1983), Lecture Notes in Math., vol. 1057, Springer, Berlin, 1984, pp. 57-105, DOI 10.1007/BFb0098594. MR753298 (86d:58084)

[13] Toby Elmhirst and Martin Golubitsky, Nilpotent Hopf bifurcations in coupled cell systems, SIAM J. Appl. Dyn. Syst. 5 (2006), no. 2, 205-251, DOI 10.1137/050635559. MR2237146 (2007m:37116)

[14] Michael Field, Lectures on bifurcations, dynamics and symmetry, Pitman Research Notes in Mathematics Series, vol. 356, Longman, Harlow, 1996. MR1425388 (97h:58115)

[15] Michael Field, Symmetry breaking for equivariant maps, Algebraic groups and Lie groups, Austral. Math. Soc. Lect. Ser., vol. 9, Cambridge Univ. Press, Cambridge, 1997, pp. 219-253. MR:1635684(99k:58127)

[16] Michael Field, Combinatorial dynamics, Dyn. Syst. 19 (2004), no. 3, 217-243, DOI 10.1080/14689360410001729379. MR2084549 (2005g:37057)

[17] Michael Field and Martin Golubitsky, Symmetry in chaos, 2nd ed., Society for Industrial and Applied Mathematics (SIAM), Philadelphia, PA, 2009. A search for pattern in mathematics, art, and nature. MR2517341 (2010j:37054)

[18] Natasha Filipski and Martin Golubitsky, The abelian Hopf $H \bmod K$ theorem, SIAM J. Appl. Dyn. Syst. 9 (2010), no. 2, 283-291, DOI 10.1137/090756582. MR.2644921 (2012d:37117)

[19] Martin Golubitsky and Carla Alves-Pinto, Bipedal locomotion, EQUADIFF 2003, World Sci. Publ., Hackensack, NJ, 2005, pp. 452-457, DOI 10.1142/9789812702067_0072. MR2185070

[20] Martin Golubitsky, Krešimir Josić, and LieJune Shiau, Bursting in coupled cell systems, Bursting, World Sci. Publ., Hackensack, NJ, 2005, pp. 201-221. MR2210829 
[21] M. Golubitsky and M. Krupa, Stability computations for nilpotent Hopf bifurcations in coupled cell systems, Internat. J. Bifur. Chaos Appl. Sci. Engrg. 17 (2007), no. 8, 2595-2603, DOI 10.1142/S0218127407018658. MR2355521 (2008i:37101)

[22] Martin Golubitsky and Reiner Lauterbach, Bifurcations from synchrony in homogeneous networks: linear theory, SIAM J. Appl. Dyn. Syst. 8 (2009), no. 1, 40-75, DOI 10.1137/070704873. MR2481276 (2010f:37083)

[23] Martin Golubitsky, Jerrold E. Marsden, Ian Stewart, and Michael Dellnitz, The constrained Liapunov-Schmidt procedure and periodic orbits, Normal forms and homoclinic chaos (Waterloo, ON, 1992), Fields Inst. Commun., vol. 4, Amer. Math. Soc., Providence, RI, 1995, pp. 81-127. MR:1350545 (96g:58135)

[24] M. Golubitsky, M. Nicol, and I. Stewart, Some curious phenomena in coupled cell networks, J. Nonlinear Sci. 14 (2004), no. 2, 207-236, DOI 10.1007/s00332-003-0593-6. MR2041431 (2005a:37037)

[25] M. Golubitsky, M. Pivato, and I. Stewart, Interior symmetry and local bifurcation in coupled cell networks, Dyn. Syst. 19 (2004), no. 4, 389-407, DOI 10.1080/14689360512331318006. MR2107649 (2006h:37041)

[26] Martin Golubitsky and Claire Postlethwaite, Feed-forward networks, center manifolds, and forcing, Discrete Contin. Dyn. Syst. 32 (2012), no. 8, 2913-2935, DOI 10.3934/dcds.2012.32.2913. MR2903993

[27] Martin Golubitsky, David Romano, and Yunjiao Wang, Network periodic solutions: full oscillation and rigid synchrony, Nonlinearity 23 (2010), no. 12, 3227-3243, DOI 10.1088/09517715/23/12/012. MR2739423 (2012k:34069)

[28] Martin Golubitsky and David G. Schaeffer, Singularities and groups in bifurcation theory. Vol. I, Applied Mathematical Sciences, vol. 51, Springer-Verlag, New York, 1985. MR.771477 (86e:58014)

[29] Martin Golubitsky, LieJune Shiau, Claire Postlethwaite, and Yanyan Zhang, The feed-forward chain as a filter-amplifier motif, Coherent behavior in neuronal networks, Springer Ser. Comput. Neurosci., vol. 3, Springer, New York, 2009, pp. 95-120, DOI 10.1007/978-1-4419-03891_6. MR2758921 (2012g:92044)

[30] Martin Golubitsky, LieJune Shiau, and Andrei Török, Symmetry and pattern formation on the visual cortex, Dynamics and bifurcation of patterns in dissipative systems, World Sci. Ser. Nonlinear Sci. Ser. B Spec. Theme Issues Proc., 12, World Sci. Publ., Hackensack, NJ, 2004, pp. 3-19, DOI 10.1142/9789812567840_0001. MR2328330(2008e:92011)

[31] Martin Golubitsky and Ian Stewart, Symmetry and pattern formation in coupled cell networks, Pattern formation in continuous and coupled systems (Minneapolis, MN, 1998), IMA Vol. Math. Appl., vol. 115, Springer, New York, 1999, pp. 65-82, DOI 10.1007/978-1-46121558-5_6. MR:1708862 (2001h:34060)

[32] Martin Golubitsky and Ian Stewart, Patterns of oscillation in coupled cell systems, Geometry, mechanics, and dynamics, Springer, New York, 2002, pp. 243-286, DOI 10.1007/0-387-217916_8. MR.1919832 (2003j:34063)

[33] Martin Golubitsky and Ian Stewart, The symmetry perspective, Progress in Mathematics, vol. 200, Birkhäuser Verlag, Basel, 2002. From equilibrium to chaos in phase space and physical space. MR1891106 (2003e:37068)

[34] Martin Golubitsky and Ian Stewart, Synchrony versus symmetry in coupled cells, EQUADIFF 2003, World Sci. Publ., Hackensack, NJ, 2005, pp. 13-24, DOI 10.1142/9789812702067_0002. MR2185000

[35] Martin Golubitsky and Ian Stewart, Nonlinear dynamics of networks: the groupoid formalism, Bull. Amer. Math. Soc. (N.S.) 43 (2006), no. 3, 305-364, DOI 10.1090/S0273-0979-06-011086. MR2223010 (2008k:37182)

[36] Martin Golubitsky, Ian Stewart, Pietro-Luciano Buono, and J. J. Collins, A modular network for legged locomotion, Phys. D 115 (1998), no. 1-2, 56-72, DOI 10.1016/S0167-2789(97)002224. MR1616780 (99d:92051)

[37] Martin Golubitsky, Ian Stewart, and David G. Schaeffer, Singularities and groups in bifurcation theory. Vol. II, Applied Mathematical Sciences, vol. 69, Springer-Verlag, New York, 1988. MR950168 (89m:58038)

[38] Martin Golubitsky, Ian Stewart, and Andrei Török, Patterns of synchrony in coupled cell networks with multiple arrows, SIAM J. Appl. Dyn. Syst. 4 (2005), no. 1, 78-100, DOI 10.1137/040612634. MR2136519(2005k:34143) 
[39] James E. Humphreys, Introduction to Lie algebras and representation theory, Graduate Texts in Mathematics, vol. 9, Springer-Verlag, New York, 1978. Second printing, revised. MR 499562 (81b:17007)

[40] Maria da Conceição A Leite and Martin Golubitsky, Homogeneous three-cell networks, Nonlinearity 19 (2006), no. 10, 2313-2363, DOI 10.1088/0951-7715/19/10/004. MR2260266 (2007m:37118)

[41] Jerrold E. Marsden and Tudor S. Ratiu, Introduction to mechanics and symmetry, 2nd ed., Texts in Applied Mathematics, vol. 17, Springer-Verlag, New York, 1999. A basic exposition of classical mechanical systems. MR1723696 (2000i:70002)

[42] James Murdock, Normal forms and unfoldings for local dynamical systems, Springer Monographs in Mathematics, Springer-Verlag, New York, 2003. MR.1941477 (2003k:37070)

[43] M. J. Parker, M. G. M. Gomes, and I. N. Stewart, Forced symmetry-breaking of square lattice planforms, J. Dynam. Differential Equations 18 (2006), no. 1, 223-255, DOI 10.1007/s10884005-9004-z. MR2220196 (2008c:37079)

[44] Carla M. A. Pinto and Martin Golubitsky, Central pattern generators for bipedal locomotion, J. Math. Biol. 53 (2006), no. 3, 474-489, DOI 10.1007/s00285-006-0021-2. MR2251782 (2007i:92009)

[45] Jan A. Sanders, Normal form theory and spectral sequences, J. Differential Equations 192 (2003), no. 2, 536-552, DOI 10.1016/S0022-0396(03)00038-X. MR.1990851 (2004c:37103)

[46] Jan A. Sanders, Normal form in filtered Lie algebra representations, Acta Appl. Math. 87 (2005), no. 1-3, 165-189, DOI 10.1007/s10440-005-1143-z. MR2151128 (2006b:37094)

[47] J. A. Sanders, F. Verhulst, and J. Murdock, Averaging methods in nonlinear dynamical systems, 2nd ed., Applied Mathematical Sciences, vol. 59, Springer, New York, 2007. MR2316999 (2008h:34001)

[48] I. Stewart, Networking opportunity, Nature 427 (2004), 601-604.

[49] Ian Stewart, The lattice of balanced equivalence relations of a coupled cell network, Math. Proc. Cambridge Philos. Soc. 143 (2007), no. 1, 165-183, DOI 10.1017/S0305004107000345. MR:2340982(2008m:37033)

[50] Ian Stewart and Martin Golubitsky, Synchrony-breaking bifurcation at a simple real eigenvalue for regular networks 1: 1-dimensional cells, SIAM J. Appl. Dyn. Syst. 10 (2011), no. 4, 1404-1442, DOI 10.1137/110825418. MR2873187

[51] Ian Stewart, Martin Golubitsky, and Marcus Pivato, Symmetry groupoids and patterns of synchrony in coupled cell networks, SIAM J. Appl. Dyn. Syst. 2 (2003), no. 4, 609-646, DOI 10.1137/S1111111103419896. MR2050244 (2005i:37030)

[52] Ian Stewart and Martyn Parker, Periodic dynamics of coupled cell networks. I. Rigid patterns of synchrony and phase relations, Dyn. Syst. 22 (2007), no. 4, 389-450, DOI 10.1080/14689360701450410. MR2377209 (2009f:37028)

[53] Yunjiao Wang and Martin Golubitsky, Two-colour patterns of synchrony in lattice dynamical systems, Nonlinearity 18 (2005), no. 2, 631-657, DOI 10.1088/0951-7715/18/2/010. MR2122678 (2005i:37032)

Department of Mathematics, VU University, Amsterdam, The Netherlands

E-mail address: b.w.rink@vu.nl

Department of Mathematics, VU University, Amsterdam, The Netherlands

E-mail address: jan.sanders.a@gmail.com 